\title{
ORTHOGONALITY AND LINEAR FUNCTIONALS IN NORMED LINEAR SPACES
}

\author{
BY \\ ROBERT C. JAMES(1)
}

The natural definition of orthogonality of elements of an abstract Euclidean space is that $x \perp y$ if and only if the inner product $(x, y)$ is zero. Two definitions have been given $[11]\left({ }^{2}\right)$ which are equivalent to this and can be generalized to normed linear spaces, preserving the property that every twodimensional linear subset contain nonzero orthogonal elements. The definition which will be used here (Definition 1.2) has the added advantage of being closely related to the theories of linear functionals and hyperplanes. The theory and applications of this orthogonality have been organized in the following sections, which are briefly outlined:

1. Fundamental definitions. An element $x$ is orthogonal to an element $y$ if and only if $\|x+k y\| \geqq\|x\|$ for all $k$. This orthogonality is homogeneous, but is neither symmetric nor additive.

2. Existence of orthogonal elements. An element $x$ of a normed linear space is orthogonal to at least one hyperplane through the origin, while for elements $x$ and $y$ there is at least one number $a$ for which $a x+y \perp x$ or $\|a x+y\|$ is minimum (Theorems 2.2-2.3).

3. Orthogonality in general normed linear spaces. The limits $N_{ \pm}(x ; y)$ $= \pm \lim _{n \rightarrow \pm \infty}\|n x+y\|-\|n x\|=\lim _{h \rightarrow \pm 0}[\|x+h y\|-\|x\|] / h$ exist and satisf $y$ weakened linearity conditions. Also, $x \perp a x+y$ if and only if $N_{-}(x ; y) \leqq-a\|x\|$ $\leqq N_{+}(x ; y)$, while $N_{+}(y ; x)=0$ for all nonzero $x$ and $y$ satisfying $N_{+}(x ; y)=0$ if and only if orthogonality is symmetric and $N_{-}(x ; y) \equiv N_{+}(x ; y)$ (Theorems 3.2 and 3.5 ).

4. Types of uniqueness of orthogonality. An element $x$ of a normed linear space is orthogonal to only one hyperplane through the origin if and only if orthogonality is additive, or if and only if the norm is Gateaux differentiable (Theorems 4.1-4.2). The space is strictly convex if and only if there is a unique number $a$ for which $a x+y \perp x$ (Theorem 4.3).

5. Hyperplanes and linear functionals. Conditions for orthogonality can be given in terms of hyperplanes, while there is a unique number $a$ with $x_{0} \perp a x_{0}+y$ if and only if there is a unique linear functional $f$ with $\|f\|=1$ and $f\left(x_{0}\right)=\left\|x_{0}\right\|$, or if and only if the sphere $\|x\| \leqq\left\|x_{0}\right\|$ has a tangent hyper-

Presented to the Society, November 27, 1943 and April 29, 1944; received by the editors April 28, 1946.

(1) The author wishes to express his appreciation of the encouragement and advice of Professor Michal.

(2) Numbers in brackets refer to the references at the end of the paper. 
plane at $x_{0}$ (Theorem 5.3) - there being analogous conditions for the uniqueness of the number $a$ for which $a x+y \perp x$. These types of uniqueness can be related by using conjugate spaces (Theorems 5.3-5.6).

6. Existence of inner products. An inner product can be defined in a normed linear space if $N_{+}(x ;\|x\| y) \equiv N_{+}(y ;\|y\| x)$ (Theorem 6.4), while this is true for a space of three or more dimensions if orthogonality is symmetric and additive, or if $N_{+}(y ; x)=0$ for all nonzero elements $x$ and $y$ satisfying $N_{+}(x ; y)=0$ (Theorems 6.1-6.2).

7. Existence of elements orthogonal to linear subsets. There is an element orthogonal to each closed linear subset of a normed linear space if and only if each linear functional takes on its maximum in the unit sphere, while various sufficient conditions for this can be given (Theorems 7.1-7.3). One of these is uniform convexity, which also implies the uniqueness of the element on a given side of a hyperplane $H$ through the origin and orthogonal to $H$ (Theorem 7.4).

8. General forms for linear functionals. If no element of a normed linear space is orthogonal to more than one hyperplane through the origin, and if there is at least one element orthogonal to each closed linear subset, then for any linear functional $f$ defined in the space and having $\|f\|=1$ there is an element $x_{0}$ for which $f(y) \equiv N\left(x_{0} ; y\right) \equiv-a\left\|x_{0}\right\|$, where $N\left(x_{0} ; y\right)$ is the Gateaux differential of the norm and $x_{0} \perp a x_{0}+y$. Other conditions for this can be given, while the element $x_{0}$ (with $\left\|x_{0}\right\|=1$ ) is unique for a given $f$ if the space is strictly convex.

1. Fundamental definitions. A normed linear space is a linear (vector) space $[2$, p. 26] whose multiplier domain is the field of real numbers and which has associated with each element $x$ a real number $\|x\|$ satisfying the postulates:

1. $\|x\|>0$ if $x \neq 0$.

2. $a x\|=|a| \cdot\| x \|$ for all numbers $a$.

3. $\|x+y\| \leqq\|x\|+\|y\|$.

It follows from (3) that the functional $\|x\|$ is continuous. A normed linear space which is complete is a Banach space [2, p. 53].

Definition 1.1. An abstract Euclidean space is a normed linear space such that to each ordered pair of elements $x$ and $y$ there can be associated a number $(x, y)$ with the following properties:

(1) $(t x, y)=t(x, y)$ for all numbers $t$.

(2) $(x, y)=(y, x)$.

(3) $(x, y+z)=(x, y)+(x, z)$.

(4) $\|x\|^{2}=(x, x)$.

The number $(x, y)$ is the (bilinear and symmetric) inner product of $x$ and $y$ [9, p. 707].

A function $f$ with real number values and argument in a normed linear space is a linear functional if it is continuous and $f(x+y)=f(x)+f(y)$ for all 
elements $x$ and $y$. It then follows that there is a number $M$ such that $|f(x)| \leqq M \cdot\|x\|$ for all elements $x$, the lower bound of such numbers $M$ being the modulus of $f[2$, p. 54]. If $\|f\|$ is the modulus of $f$, then the set of all linear functionals defined on a normed linear space $T$ is also a normed linear spacethe conjugate space $T^{\prime}$.

The definition of orthogonality " $x \perp y$ if and only if $\|x+k y\|=\|x-k y\|$ identically in $k$ " was suggested by Roberts [18, p. 56], but has the weakness that for some normed linear spaces at least one of every pair of orthogonal elements would have to be zero [11, Example 2.1]. This difficulty is not encountered in either of the definitions " $x \perp y$ if and only if $\|x+y\|=\|x-y\|$ " or " $x \perp y$ if and only if $\|x\|^{2}+\|y\|^{2}=\|x-y\|^{2}$ " used in a previous paper [11], nor in the following definition.

Definition 1.2. An element $x$ of a normed linear space is orthogonal to an element $y(x \perp y)$ if and only if $\|x+k y\| \geqq\|x\|$ for all $k\left({ }^{3}\right)$. An element $x$ is orthogonal to a subset $H$ if and only if $x \perp h$ for all $h \in H$.

This is the analogy of: If two lines $L_{1}$ and $L_{2}$ intersect at $p$, then $L_{1} \perp L_{2}$ if and only if the distance from a point of $L_{2}$ to a given point $q$ of $L_{1}$ is never less than the distance from $p$ to $q$. It is clear that if $x$ is orthogonal to $x$, then $x$ is zero. This definition has the advantage that it can be related to other concepts, such as strict convexity, weak compactness, linear functionals, and hyperplanes. This will largely be done by means of the following properties of orthogonality in ordinary Euclidean spaces.

I. SYMmetry. If $x \perp y$, then $y \perp x$.

II. HoMogeneity. If $x \perp y$, then $a x \perp b y$ for all numbers $a$ and $b$.

III. AdDitivity. If $x \perp y$ and $x \perp z$, then $x \perp(y+z)$.

IV. For any elements $x$ and $y$ there exists a number a such that $x \perp(a x+y)$.

The last property is important as an assurance that a concept of orthogonality is not trivial. An even stronger condition is satisfied by the orthogonality of Definition 1.2 (Theorem 2.2). This orthogonality is clearly homogeneous, but is not necessarily symmetrical nor additive (Theorems 4.2 and 6.1).

For abstract Euclidean spaces, $\|x+k y\|^{2}=\|x\|^{2}+2 k(x, y)+k^{2}\|y\|^{2}$. Hence $x \perp y$ if and only if $(x, y)=0$. The orthogonality of Definition 1.2 is therefore symmetric and additive in abstract Euclidean spaces. Also, $(x, a x+y)$ $=a\|x\|^{2}+(x, y)$, and $x \perp a x+y$ if and only if $a=-(x, y) /\|x\|^{2}$.

2. Existence of orthogonal elements.

Definition 2.1. A hyperplane of a normed linear space $T$ is any proper closed linear subset $M$ which is not properly contained in a proper linear subset of $T$, or any translation $x+M$ of such a linear subset $M$.

Clearly if $\left\{y_{n}\right\}$ is a sequence converging to $y$, and $x$ is orthogonal to each $y_{n}$, then $x \perp y$. Hence for any $x$ the set of all $y$ with $x \perp y$ is closed. For any element $x$ of a normed linear space there is at least one hyperplane $H$ through the origin with $x \perp H$, the uniqueness of $H$ being equivalent to Gateaux differ-

(3) This definition was used by Birkhoff [3] and by Fortet [ 6 and 7$]$. 
entiability of the norm and to additivity of orthogonality ( $\$ 4)$. The following theorems are basic to much that follows.

THEOREM 2.1. If $f(\neq 0)$ is a linear functional on a normed linear space $T$, then $|f(x)|=\|f\| \cdot\|x\|$ if and only if $x \perp H$, where $H$ is the hyperplane of all $h$ for which $f(h)=0$. If $x$ is orthogonal to a linear subset $L \subset T$, then there exists a linear functional $f$ for which $f(x)=\|f\| \cdot\|x\|$ and $f(y)=0$ if $y \in L$, and there is a hyperplane $H$ through the origin with $x \perp H$ and $L \subset H$.

Proof. Let $H$ be the hyperplane consisting of all elements $h$ for which $f(h)=0$. Suppose $|f(x)|=\|f\| \cdot\|x\|$. Then $f(h)=0$ implies $|f(x+h)|=|f(x)|$ $\leqq\|f\| \cdot\|x+h\|$, and hence that $\|x+h\| \geqq\|x\|$ for all elements $h \in H$. That is, $x \perp H$. Conversely, suppose $x \perp H$ and $|f(x)|=p\|x\|$. But $\|x\| \leqq\|x+h\|$ if $h \in H$, and hence $|f(x+h)|=|f(x)|=p\|x\| \leqq p\|x+h\|$. Since $H$ is a hyperplane through the origin, it follows that $|f(y)| \leqq p\|y\|$ for all $y$ of $T$. That is, $p=\|f\|$ and $|f(x)|=\|f\| \cdot\|x\|$. The remainder of the theorem follows from a known property of linear functionals [2, p. 57 , lemma].

The following theorem is a direct consequence of Theorem 2.1 and the fact that for any element $x$ of a normed linear space there is a linear functional $f$ such that $f(x)=\|f\| \cdot\|x\|[2$, p. 55, Theorem 3].

ThEOREM 2.2. Any element of a normed linear space is orthogonal to some hyperplane through the origin.

However, for a given hyperplane $H$ through the origin there does not necessarily exist an element $x$ with $x \perp H$. This problem is equivalent to finding an element $x$ with $f(x)=\|f\| \cdot\|x\|$ for a given linear functional $f$, and will be studied in $\$ 7$.

CoROllaRy 2.2. If $x$ and $y$ are any two elements of a normed linear space, then there exists a number a such that $x \perp a x+y$. Also, $x \perp a x+y$ if and only if there is a linear functional $f$ with $|f(x)|=\|f\| \cdot\|x\|$ and $a=-f(y) / f(x)$. If $x \perp a x+y$, then $|a| \leqq\|y\| /\|x\|$.

Proof. The first parts of the corollary follow from Theorems 2.1 and 2.2. By definition, $x \perp a x+y$ if and only if $\|x+k(a x+y)\| \geqq\|x\|$ for all $k$. Letting $k=-1 / a$ gives $|a| \leqq\|y\| /\|x\|$.

For vectors $x$ and $y$ in ordinary Euclidean space, $x \perp a x+y$ if $a=-\cos \theta\|y\| /\|x\|$, where $\theta$ is the angle between $x$ and $y$. In that case, $\|x+k(a x+y)\|>\|x\|$ if $k \neq 0$. However, for elements $x$ and $y$ of a normed linear space it is possible to satisfy this stronger inequality if and only if the space is strictly convex (Theorems 2.3 and 4.3 ).

Unlike other types of orthogonality which have been used [11 and 18], $x \perp y$ does not imply $y \perp x$. Thus Corollary 2.2 does not give the existence of a number $b$ such that $b x+y \perp x$. Likewise, the uniqueness of the number $a$ with $x \perp a x+y$ is independent of that of the number $b$ with $b x+y \perp x$. The differ- 
ence in these two conditions is partially evident from the following theorem, and will be shown clearly later (\$4).

THEOREM 2.3. For any elements $x$ and $y$ of a normed linear space, there exists a number $a$ such that $a x+y \perp x$. This number $a$ is a value of $k$ for which $\|k x+y\|$ takes on its absolute minimum. If $A x+y \perp x$ and $B x+y \perp x$, then $a x+y \perp x$ if $a$ is $a$ number between $A$ and $B$.

Proof. By definition, $a x+y \perp x$ if and only if $\|(a x+y)+k x\| \geqq\|a x+y\|$ for all $k$, or if and only if $\|a x+y\|$ is the smallest value of $\|k x+y\|$. Since $\|k x+y\|$ is continuous in $k$, it must take on its minimum. Suppose $A x+y \perp x$ and $B x+y \perp x$. Since the function $f(n)=\|x+n y\|$ is convex (except for possible straight line segments), it follows that $\|a x+y\|=\|A x+y\|=\|B x+y\|$ if $a$ is between $A$ and $B$, and that $x \perp a x+y$.

Let $a$ and $b$ be numbers such that $x \perp a x+y$ and $y \perp b y+x$. If $x$ and $y$ are elements of an abstract Euclidean space, then $b\|y\|^{2}=a\|x\|^{2}$. Also, $a b\|x\|^{2}\|y\|^{2}=(x, y)^{2}$, or $\cos ^{2} \theta=a b$, where $\theta$ is the angle between $x$ and $y$. In a normed linear space it is possible to have $a b=1$ without $x$ and $y$ being parallel, but the following generalization is possible.

THEOREM 2.4. If $x$ and $y$ are any two nonzero elements of a normed linear space, then $|a b| \leqq 1$ if $x \perp a x+y$ and $y \perp b y+x$. If orthogonality is symmetric, then $0 \leqq a b \leqq 1$.

Proof. By Corollary 2.2, $|a| \leqq\|y\| /\|x\|$ and $|b| \leqq\|x\| /\|y\|$. Hence $|a b| \leqq 1$. If orthogonality is symmetric and $x \perp a x+y$, then $a x+y \perp x$ and $\|(a x+y)$ $+k_{1} x\|\geqq\| a x+y \|$ for all $k_{1}$. If $y \perp b y+x$, then $\left\|y+k_{2}(b y+x)\right\| \geqq\|y\|$ for all $k_{2}$. Letting $k_{1}=-a$ gives $\|y\| \geqq\|a x+y\|$, and $k_{2}=a /(1-a b)$ gives $\|a x+y\|$ $\geqq|1-a b|\|y\|$. Hence $\|y\| \geqq|1-a b|\|y\|$ and $a b \geqq 0$.

3. Orthogonality in general normed linear spaces. For abstract Euclidean spaces, $\lim _{n \rightarrow \infty}\|n x+y\|-\|n x\|$, or $N_{+}(x ; y)=\lim _{h \rightarrow+0}[\|x+h y\|-\|x\|] / h$, is zero if and only if $x \perp y$. While this is not true in normed linear spaces, this limit is closely related to orthogonality and its being zero implies $x \perp y$. These limits are known to exist $\left({ }^{4}\right)$, but the proof of their existence and an evaluation of the limits in terms of orthogonality can be given simultaneously.

TheOREM 3.1. For any elements $x(\neq 0)$ and $y$ of a normed linear space, $\lim _{n \rightarrow \infty}\|n x+y\|-\|n x\|=-A\|x\|$ and $\lim _{n \rightarrow \infty}\|n x\|-\|n x-y\|=-B\|x\|$, where $A$ and $B$ are the algebraically smallest and largest of the numbers a such that $x \perp a x+y$.

Proof. Since $|\|n x+y\|-\|n x\|| \leqq\|y\|$ for all $n,\|n x+y\|-\|n x\|$ has a limit point as $n$ becomes infinite. Let $-r\|x\|$ be such a limit point and $\left\{n_{i}\right\}$ be a sequence of numbers such that $\lim _{i \rightarrow \infty} n_{i}=\infty$ and $\lim _{i \rightarrow \infty}\left\|n_{i} x+y\right\|-\left\|n_{i} x\right\|$ $=-r\|x\|$. It is known [11, Lemma 4.4] that for any number $a$ this limit

(4) See Mazur [14, p. 75], or Taylor [22, Theorem 5.21]. 
is equal to $\lim _{i_{i} \infty}\left\|n_{i} x+(a x+y)\right\|-\left\|n_{i} x\right\|-a\|x\|$. If $x \perp a x+y$, then $\| n_{i} x$ $+(a x+y)\|\geqq\| n_{i} x \|$, and hence $\lim _{i \rightarrow \infty}\left\|n_{i} x+y\right\|-\left\|n_{i} x\right\| \geqq-a\|x\|$. Thus if $A$ is the greatest lower bound of all numbers $a$ for which $x \perp a x+y$, then

$$
\lim _{i \rightarrow \infty}\left\|n_{i} x+y\right\|-\left\|n_{i} x\right\| \geqq-A\|x\| \text {. }
$$

Let $\epsilon$ be any positive number, and choose $N$ such that $\mid\left\|n_{i} x+y\right\|-\left\|n_{i} x\right\|$ $+r\|x\| \mid<\epsilon\|x\|$ if $n_{i}>N$. If $n_{i}>|r|$, this can be written $\left|\left\|n_{i} x+y\right\|-\left\|\left(n_{i}-r\right) x\right\|\right|$ $<\epsilon\|x\|_{.}$. There is then a number $e_{i}$ such that $\left|e_{i}\right|<\epsilon$ and $\left\|n_{i} x+y\right\|=\|\left(n_{i}-r\right.$ $\left.+e_{i}\right) x \|$. But this can be written

$$
\begin{aligned}
\|\left[\left(n_{i}-2^{-1} r\right.\right. & \left.\left.+2^{-1} e_{i}\right) x+2^{-1} y\right]+\left[\left(2^{-1} r-2^{-1} e_{i}\right) x+2^{-1} y\right] \| \\
& =\left\|\left[\left(n_{i}-2^{-1} r+2^{-1} e_{i}\right) x+2^{-1} y\right]-\left[\left(2^{-1} r-2^{-1} e_{i}\right) x+2^{-1} y\right]\right\| .
\end{aligned}
$$

It then follows that for $|k| \geqq 1[11$, Theorem 4.1]

$$
\begin{aligned}
&\left\|\left[\left(n_{i}-2^{-1} r+2^{-1} e_{i}\right) x+2^{-1} y\right]+k\left[\left(2^{-1} r-2^{-1} e_{i}\right) x+2^{-1} y\right]\right\| \\
& \geqq\left\|\left(n_{i}-2^{-1} r+2^{-1} e_{i}\right) x+2^{-1} y\right\|
\end{aligned}
$$

If $k$ is replaced by $2 m$ and this inequality divided by $|m|$, it becomes

$$
\left\|\left[\frac{\left(2 n_{i}-r+e_{i}\right) x+y}{2 m}\right]+\left(r-e_{i}\right) x+y\right\| \geqq\left\|\frac{\left(2 n_{i}-r+e_{i}\right) x+y}{2 m}\right\| .
$$

Since $\left|e_{i}\right|<\epsilon$, it follows that $\lim _{i \rightarrow \infty} e_{i}=0$. Hence if $p$ is any number and $n_{i}$ becomes infinite, but with $m=n_{i} / p$, it follows that $\|p x+(r x+y)\| \geqq\|p x\|$ for all $p$. Thus if $-r\|x\|$ is a limit point of $\|n x+y\|-\|n x\|$ as $n$ becomes infinite, then $x \perp r x+y$. But it was also shown that $-r\|x\| \geqq-A\|x\|$, or $A \geqq r$. It therefore follows that $r=A, \lim _{n \rightarrow \infty}\|n x+y\|-\|n x\|$ exists and equals $-A\|x\|$, and that $x \perp A x+y$. If $B$ is the largest number such that $x \perp B x+y$, then $-B$ is the smallest number for which $x \perp-B x-y$. Hence $\lim _{n \rightarrow \infty}\|n x\|-\|n x-y\|$ $=-B\|x\|$.

LEMma 3.1. If $x$ and $y$ are elements of a normed linear space and $x \perp A x+y$ and $x \perp B x+y$, then $x \perp a x+y$ if $a$ is a number between $A$ and $B$.

Proof. For definiteness, assume $A<B$. If $x \perp A x+y$ and $x \perp B x+y$, then $\|x+k(A x+y)\| \geqq\|x\|$ and $\|x+k(B x+y)\| \geqq\|x\|$ for all $k$. Let $a$ be any number for which $A \leqq a \leqq B$. If $k \geqq 0$, then

$$
\begin{aligned}
\|x+k(a x+y)\| & =\|[1+k(a-A)] x+k(A x+y)\| \\
& \geqq\|[1+k(a-A)] x\| \geqq\|x\| .
\end{aligned}
$$

If $k \leqq 0$, then

$$
\begin{aligned}
\|x+k(a x+y)\| & =\|[1+k(a-B)] x+k(B x+y)\| \\
& \geqq\|[1+k(a-B)] x\| \geqq\|x\| .
\end{aligned}
$$


Hence $\|x+k(a x+y)\| \geqq\|x\|$ for all $k$, and $x \perp a x+y$.

THEOREM 3.2. If $x$ and $y$ are any two elements of a normed linear space, then $x \perp a x+y$ if and only if

$$
-\lim _{n \rightarrow \infty}\|n x+y\|-\|n x\| \leqq a\|x\| \leqq-\lim _{n \rightarrow \infty}\|n x\|-\|n x-y\| .
$$

The usefulness of this theorem in determining the numbers $a$ for which $x \perp a x+y$ can be shown by using the Banach space of number pairs $(a, b)$, with $\|(a, b)\|=\left(a^{2}+b^{2}+|a b|\right)^{1 / 2}$. If $x=(1,0)$ and $y=(0,1)$, then $n x+y=(n, 1)$ and $n x-y=(n,-1)$. From Theorem 3.2, $x \perp a x+y$ if and only if $-1 / 2 \leqq a$ $\leqq 1 / 2$. That is, $(1,0) \perp(a, 1)$ if and only if $-1 / 2 \leqq a \leqq 1 / 2$. This is equivalent to saying that $\left[(1+k a)^{2}+k^{2}+|k(1+k a)|\right]^{1 / 2} \geqq 1$ for all $k$ if and only if $|a| \leqq 1 / 2$.

If the two limits of Theorem 3.1 are added, it is seen that the number $\alpha$ defined by $\lim _{n \rightarrow \infty}\|n x+y\|-\|n x-y\|=-2 \alpha\|x\|$ is the mean of the largest and smallest of all numbers $a$ for which $x \perp a x+y$. The following theorem follows from this and the fact that $\lim _{n \rightarrow \infty}\|n x+(a x+y)\|-\|n x+y\|=a\|x\|[11$, Lemma 4.4].

THEOREM 3.3. If $\lim _{n \rightarrow \infty}\|n x+(\alpha x+y)\|-\|n x-(\alpha x+y)\|=0$ for elements $x$ and $y$ of a normed linear space, then $x \perp \alpha x+y$ and $\alpha=2^{-1}(A+B)$, where $A$ and $B$ are the smallest and largest of the numbers a for which $x \perp a x+y$.

It is known that for any elements $x$ and $y$ of a normed linear space there is a number $a$ for which $\|x+(a x+y)\|=\|x-(a x+y)\|$, and that, for such numbers $a,\|x+k(a x+y)\|>2^{-1}\|x\|$ for all $k$ [11, Theorems 4.4 and 4.2]. This could be used as a definition of orthogonality, but it is clear that $x$ and $a x+y$ satisfying this equality are not necessarily orthogonal in the present sense (Definition 1.2). However, it is possible to relate these types of orthogonality:

THEOREM 3.4. If $x$ and $y$ are any two elements of a normed linear space, and the numbers $\alpha_{n}$ are such that

$$
\left\|x+n^{-1}\left(\alpha_{n} x+y\right)\right\|=\left\|x-n^{-1}\left(\alpha_{n} x+y\right)\right\|,
$$

then $\lim _{n \rightarrow \infty} \alpha_{n}=\alpha$ exists and $x \perp \alpha x+y$. Furthermore, $\alpha$ is the mean of the largest and smallest of the numbers a for which $x \perp a x+y$.

Proof. The numbers $\alpha_{n}$ are bounded as $n$ becomes infinite [11, Theorem 4.5], and hence have a limit point $\alpha$. It then follows from $\left\|n x+\left(\alpha_{n} x+y\right)\right\|$ $-\left\|n x-\left(\alpha_{n} x+y\right)\right\|=0$ that $\lim _{n \rightarrow \infty}\|n x+(\alpha x+y)\|-\|n x-(\alpha x+y)\|=0$. Thus $\alpha$ is the mean of the largest and smallest of the numbers $a$ for which $x \perp a x+y$ (Theorem 3.3), and $\lim _{n \rightarrow \infty} \alpha_{n}=\alpha$.

The norm of a general normed linear space is not differentiable at all nonzero points, although $\lim _{h \rightarrow+0}(\|x+h y\|-\|x\|) / h$ exists. If this limit is denoted by $N_{+}(x ; y)$, and $\lim _{h \rightarrow-0}(\|x+h y\|-\|x\|) / h$ by $N_{-}(x ; y)$, then, if $x \neq 0$, 


$$
N_{+}(x ; y)=-A\|x\| \quad \text { and } \quad N_{-}(x ; y)=-B\|x\|,
$$

where $A$ and $B$ are the smallest and largest of the numbers $a$ for which $x \perp a x+y$ (Theorem 3.1). If the Gateaux differential of the norm (Définition 4.3) exists at $x$, then $N_{+}(x ; y)$ is a linear functional in $y$. It is not linear in general (Example 8.1), but does satisfy the following weakened linearity conditions:

$$
\begin{aligned}
N_{+}(x ; y+z) & \leqq N_{+}(x ; y)+N_{+}(x ; z) . \\
N_{+}(x ; t y) & =t \cdot N_{+}(x ; y) \quad \text { for } t \geqq 0 . \\
N_{+}(x ; x) & =\|x\| ; \quad\left|N_{+}(x ; y)\right| \leqq\|y\| .
\end{aligned}
$$

These three relations follow from results given by Ascoli [1, pp. 53-55], but follow easily from the theory of orthogonality. The first is a direct application of the triangular inequality of the norm, while (3) follows from $-N_{+}(x ; t y) /\|x\|$ and $-N_{+}(x ; y) /\|x\|$ being the smallest of the numbers $a$ and $b$, respectively, for which $x \perp a x+t y$ and $x \perp b x+y$. The inequality (4) follows from $x \perp-\left[N_{+}(x ; y) /\|x\|\right] x+y$ and Corollary 2.2.

If $A$ is the smallest of the numbers $a$ for which $x \perp a x+s y$, then $A-r$ is the smallest of the numbers $b$ for which $x \perp b x+(r x+s y)$. Using this, it follows that:

$$
N_{+}(x ; r x+s y)=r\|x\|+s \cdot N_{+}(x ; y) \text {, }
$$

for $\mathrm{s} \geqq 0$ and all $r$.

If $x$ is an element of a normed linear space and $f$ is any linear functional with $f(x)=\|x\|$ and $\|f\|=1$, then $N_{-}(x ; y) \leqq f(y) \leqq N_{+}(x ; y)$ for all $y$; if $N_{-}(x ; y) \leqq a \leqq N_{+}(x ; y)$, then there is a linear functional $F$ for which $F(y)=a$, $F(x)=\|x\|$, and $\|F\|=1[14$, p. 75$]$. The following is an equivalent statement of this in terms of orthogonality $(5)$, the validity of which follows from the evaluation of $N_{+}(x ; y)$ and $N_{-}(x ; y)$ given by (1) above:

$$
x \perp a x+y \text { if and only if } N_{-}(x ; y) \leqq-a\|x\| \leqq N_{+}(x ; y) .
$$

If $N_{+}(x ; y)=N_{+}(y ; x)=0$, then it follows from (6) that $x \perp y$ and $y \perp x$. For general normed linear spaces neither $N_{+}(x ; y)=0$ nor $N_{+}(y ; x)=0$ is a necessary condition for the orthogonality of $x$ and $y$, while the assumption that one follows from the other is very severe (Theorems 3.5 and 6.2).

THEOREM 3.5. A necessary and sufficient condition that $N_{+}(y ; x)=0$ for any nonzero elements $x$ and $y$ of a normed linear space $T$ satisfying $N_{+}(x ; y)=0$ is that orthogonality be symmetric in $T$ and for any elements $x(\neq 0)$ and $y$ there be a unique number a for which $x \perp a x+y$.

Proof. If for all $x(\neq 0)$ and $y$ there is a unique number $a$ for which $x \perp a x+y$, then it follows from (6) that $x \perp y$ if and only if $N_{+}(x ; y)=0$. Hence if orthogonality is symmetric and $x$ and $y$ are not zero, then $N_{+}(y ; x)=0$ if

(5) The equivalence follows from Theorem 2.1, if we use the equations (1). 
and only if $N_{+}(x ; y)=0$. Now take $x \neq 0$ and $x \perp y$. Let $A$ and $B$ be such that $x \perp a x+y$ if and only if $A \leqq a \leqq B$, where $A \leqq 0 \leqq B$ (see (6) above). Then $a=0$ is the smallest value of $a$ for which $x \perp(A+a) x+y$, or $x \perp a x+(A x+y)$. Thus from (6), $N_{+}(x ; A x+y)=0$. If $A x+y=0$, then $A x+y \perp x$. If $A x+y \neq 0$, then the assumption that $N_{+}(A x+y ; x)=0$ implies $A x+y \perp x$. Likewise, $a=0$ is the largest value of $a$ for which $x \perp(B+a) x+y$, or $x \perp a x+(B x+y)$. Therefore $N_{-}(x ; B x+y)=-N_{+}(x ;-B x-y)=0$ and $N_{+}(-B x-y ; x)=0$ if $B x+y \neq 0$. Hence $B x+y \perp x$. Since $A \leqq 0 \leqq B$ and $A x+y \perp x$ and $B x+y \perp x$, it follows that $y \perp x$ (Theorem 2.3). Thus orthogonality is symmetric if $N_{+}(y ; x)=0$ for all nonzero elements $x$ and $y$ satisfying $N_{+}(x ; y)=0$. Now suppose there are two elements $x(\neq 0)$ and $y$ for which $x \perp a x+y$ if $A^{\prime} \leqq a \leqq B^{\prime}$, where $A^{\prime} \neq B^{\prime}$. Then because of symmetry, $a x+y \perp x$ and $\|a x+y\|$ is minimum and constant for $A^{\prime} \leqq a \leqq B^{\prime}$ (Theorem 2.3). If $A^{\prime}<\alpha<B^{\prime}$, then $\|\alpha x+y+h x\|-\|\alpha x+y\|=0$ if $A^{\prime}<\alpha \pm|h|<B^{\prime}$. Therefore $N_{+}(\alpha x+y ; x)=N_{-}(\alpha x+y ; x)=0$. Hence if we use the assumption of the theorem (or trivially if $\alpha x+y=0$ ) it follows that $N_{+}(x ; \alpha x+y)=N_{-}(x ; \alpha x+y)=0$. But with (6) above this is impossible if $A^{\prime} \neq B^{\prime}$. Hence for any elements $x(\neq 0)$ and $y$ there is a unique number $a$ for which $x \perp a x+y$.

4. Types of uniqueness of orthogonality. For any elements $x$ and $y$ of a normed linear space, there is a number $a$ for which $x \perp a x+y$ and a number $b$ for which $b x+y \perp x$. These numbers are not unique in general, their uniqueness being related to the concepts of differentiability of the norm and strict convexity (Theorems 4.1 and 4.3). Since orthogonality is not symmetric, this uniqueness can take the following forms:

Definition 4.1. Orthogonality is right-unique if and only if for no elements $x(\neq 0)$ and $y$ there is more than one number $a$ for which $x \perp a x+y$.

Definition 4.2. Orthogonality is left-unique if and only if for no elements $x(\neq 0)$ and $y$ there is more than one number $a$ for which $a x+y \perp x$.

If orthogonality is symmetric, then it is right-unique if and only if it is left-unique. This was the case for the or thogonality conditions $\|x+y\|=\|x-y\|$ and $\|x\|^{2}+\|y\|^{2}=\|x-y\|^{2}$ studied in a previous paper [11], but not for the orthogonality of Definition 1.2. Moreover, it is clear from (6) of $\$ 3$ that $N_{+}(x ; y)=N_{-}(x ; y)$ for all $x(\neq 0)$ and $y$ if and only if there is a unique number $a$ for which $x \perp a x+y$.

Definition 4.3. A functional $f$ defined on a normed linear space $T$ is Gateaux differentiable at a point $x$ if and only if $\lim _{h \rightarrow 0}[f(x+h y)-f(x)] / h$ exists for all elements $y$ of $T$. If this limit exists, it is the Gateaux differential at $x$, written $f(x ; y)$.

The Gateaux differential $N(x ; y)$ of the norm of an abstract Euclidean space exists at all nonzero points and is equal to $(x, y) /\|x\|$. But $x \perp y$ if and only if $(x, y)=0$. Hence $x \perp a x+y$ if and only if $N(x ; a x+y)=0$, or $N(x ; y)$ $=-a\|x\|$. This can be extended to normed linear spaces whose norm is Gateaux differentiable, since in such spaces $N_{+}(x ; y)=N_{-}(x ; y)=-a\|x\|$. 
THEOREM 4.1. Orthogonality is right-unique in a normed linear space $T$ if and only if the norm of $T$ possesses a Gateaux differential at each nonzero point. If this differential of the norm, $N(x ; y)$, exists at a point $x$, then $N(x ; y)=-a\|x\|$, where $a$ is the number for which $x \perp a x+y$.

It is known that $N(x ; y)$ is a linear functional of $y$ if it exists, from which it follows that $x \perp y$ and $x \perp z$ imply $x \perp y+z\left(^{6}\right)$. The converse of this is also true.

THEOREM 4.2. Orthogonality is additive in a normed linear space if and only if it is right-unique, or if and only if the norm is Gateaux differentiable at each nonzero point.

Proof. If orthogonality is right-unique and $x \perp y$ and $x \perp z$, then $y$ and $z$ both belong to the hyperplane $H$ through the origin for which $x \perp H$ (Theorem 2.2), $H$ being unique if for all $y$ there is a unique number $a$ for which $x \perp a x+y$. Then $y+z \in H$ and $x \perp y+z$. Conversely, suppose orthogonality is additive and $x \perp a x+y$ and $x \perp b x+y$. Then $x \perp-(b x+y)$ and additivity gives $x \perp(a-b) x$. But this means that $\|x+k(a-b) x\| \geqq\|x\|$, which is true for all $k$ only if $a=b$. Therefore orthogonality is right-unique if it is additive. That orthogonality is additive if and only if the norm is Gateaux differentiable at all nonzero points now follows from Theorem 4.1.

If the norm of a normed linear space has a Gateaux differential $N(x ; y)$ at each nonzero point $x$, then $x \perp y$ if and only if $N(x ; y)=0$, and the points $y$ satisfying $N(x ; y)=0$ form the unique hyperplane $H$ for which $x \perp H$. If $N(x ; y)$ exists, then $N(x ; y)+N(x ; z)=N(x ; y+z)$ and $x \perp y$ and $x \perp z$ imply $x \perp y+z$.

For any elements $x$ and $y$ of a normed linear space and any number $h$ there is a number $\alpha_{h}$ such that $\left\|x+h\left(\alpha_{h} x+y\right)\right\|=\left\|x-h\left(\alpha_{h} x+y\right)\right\|$ (see [11, Theorem 4.4]). Then $\lim _{h \rightarrow 0}[\|x+h(\alpha x+y)\|-\|x-h(\alpha x+y)\|] / h=0$ if and only if $\lim _{h \rightarrow 0} \alpha_{h}=\alpha$, and $\alpha$ is the mean of the largest and smallest of the numbers $a$ for which $x \perp a x+y$ (Theorems 3.3-3.4). Also, $x \perp \alpha x+y$ and $\lim _{h \rightarrow 0}[\|x+h y\|$ $-\|x-h y\|] / h=-2 \alpha\|x\|$.

If orthogonality is right-unique or additive, or if the norm is differentiable at nonzero points, then $\alpha$ is the unique number for which $x \perp \alpha x+y$, and $N(x ; \alpha x+y)=0$. While orthogonality is right-unique if and only if the norm is Gateaux differentiable at nonzero points, this does not follow for leftuniqueness unless orthogonality is symmetric. In fact, it will be shown that orthogonality is left-unique if and only if the space is strictly convex.

Definition 4.4. A normed linear space is strictly convex $\left({ }^{7}\right)$ if and only if

(6) See [15, pp. 129-130]; the Gateaux differential of the norm is always a weak differential [15] of the norm. Conditions for additivity of orthogonality have been given by Fortet [7, Lemma 3], but he unnecessarily assumes uniform convexity (Definition 5.4) as well as differentiability (regularity) of the norm.

(7) See Clarkson [4]. Strictly normalized is sometimes used [19]. 
$\|x\|+\|y\|=\|x+y\|$ and $y \neq 0$ imply the existence of a number $t$ for which $x=t y$.

The triangular inequality of the norm implies that the function $f(n)$ $=\|x+n y\|$ of the real variable $n$ is concave up except for possible straight line segments. Hence if the norm is Gateaux differentiable, then $N(x+n y ; y)$ $=f^{\prime}(n)$ is monotonic increasing and therefore continuous [22, Theorem 4.32]. It is equal to $-a_{n}\|x+n y\|$, where $x+n y \perp a_{n}(x+n y)+y$. If the normed linear space is also strictly convex, then $f(n)=\|x+n y\|$ is convex and $f^{\prime}(n)$ is a continuous, increasing function. If $x+a y \perp y$ and $x+b y \perp y$, then $\|x+a y\|$ $=\|x+b y\|$, since each is minimum. Also, $\|x+n y\|$ is constant for $a \leqq n \leqq b$ (Theorem 2.3). Therefore orthogonality is left-unique in strictly convex spaces. The converse of this is also true.

THEOREM 4 s. A necessary and sufficient condition that a normed linear space $T$ be strictly convex is that orthogonality be left-unique.

Proof. As shown above, orthogonality is left-unique in a strictly convex space. Conversely, if $T$ is not strictly convex then there are elements $x$ and $y \quad(y \neq 0)$ such that $\|x\|+\|y\|=\|x+y\|$ and $x \neq t y$ for any $t$. From $x+y=[(1-k) x+k y]+k x+(1-k) y$, it follows that $\|x+y\| \leqq\|(1-k) x+k y\|$ $+k\|x\|+(1-k)\|y\|$ if $0 \leqq k \leqq 1$. Since $\|x\|+\|y\|=\|x+y\|$, this gives $\|(1-k) x$ $+k y\|\geqq(1-k)\| x\|+k\| y \|$. But then $\|(1-k) x+k y\| \leqq(1-k)\|x\|+k\|y\|$. Hence

$$
\|(1-k) x+k y\|=(1-k)\|x\|+k\|y\|
$$

for $0 \leqq k \leqq 1$. If $k=\|x\| /(\|x\|+\|y\|)$, this becomes $\|(\|y\| x+\|x\| y)\|=2\|x\|\|y\|$. Setting $x^{\prime}=\|y\| x+\|x\| y$ and $y^{\prime}=\|y\| x-\|x\| y$, it follows that $\left\|x^{\prime}+y^{\prime}\right\|$ $=\left\|x^{\prime}-y^{\prime}\right\|=\left\|x^{\prime}\right\|$. Therefore $\left\|x^{\prime}+a y^{\prime}\right\|=\left\|x^{\prime}\right\|$ if $|a| \leqq 1$ and $\left\|x^{\prime}+a y^{\prime}\right\| \geqq\left\|x^{\prime}\right\|$ if $|a| \geqq 1$. Hence $x^{\prime}+a y^{\prime} \perp y^{\prime}$ if $|a| \leqq 1$. Since $y^{\prime}=0$ implies $x=(\|x\| /\|y\|) y$, it follows that orthogonality is not left-unique in a space not strictly convex. Hence a normed linear space is strictly convex if orthogonality is left-unique.

It is interesting to analyze the meaning of right- and left-uniqueness of orthogonality in the Banach space $C$ of continuous functions defined in $(0,1)$, where $\|f\|=\max _{(0,1)}|f(x)|[2$, p. 11]. This will give an easy means for constructing normed linear spaces in which orthogonality is right-unique, or left-unique, or both.

THEOREM 4.4. Orthogonality is left-unique in a linear subset $T$ of $C$ (or $T$ is strictly convex) if and only if all nonzero functions belonging to $T$ whose absolute values take on their maximum at a common point are multiples of each other.

Proof. If there are nonzero functions $f$ and $g$ for which $|f|$ and $|g|$ are both maximum at some point $a$, then either $\|f+g\|=|f(a)+g(a)|$ or $\|f-g\|$ $=|f(a)-g(a)|$. Hence $\|f\|+\|g\|$ is either equal to $\|f+g\|$ or to $\|f-g\|$. If $T$ is strictly convex (or orthogonality is left-unique), then there is a number $t$ 
such that $f=t g$. Conversely, if $T$ is not strictly convex, then there exist nonzero functions $f$ and $g$ such that $\|f\|+\|g\|=\|f+g\|$ and $f \neq g$ for any number $t$. Suppose $\|f\|=|f(a)|,\|g\|=|g(b)|$, and $\|f+g\|=|f(c)+g(c)|$. Then $|f(c)+g(c)|$ $=|f(a)|+|g(b)|$. But $|f(c)+g(c)| \leqq|f(c)|+|g(c)|,|f(c)| \leqq|f(a)|$, and $|g(c)|$ $\leqq|g(b)|$. Hence $|f(c)|=|f(a)|$ and $|g(c)|=|g(b)|$. That is, both $|f|$ and $|g|$ take on their maximum at $c$, even though $f$ and $g$ are not multiples of each other.

This theorem enables one to determine easily whether orthogonality is left-unique in a linear subset $T$ of the space $C$ (or whether $T$ is strictly convex). It is seen from (6) of $\S 3$ that a condition for orthogonality being rightunique in $T$ (or the norm of $T$ being differentiable) would result from an evaluation of the limits $N_{+}(x ; y)$ and $N_{-}(x ; y)$. These limits are known to exist in general normed linear spaces. For the space $C$,

$$
N_{+}(f ; g)=\lim _{h \rightarrow+0} \frac{\max |f+h g|-\max |f|}{h} .
$$

It follows from results of Fréchet [8] that the norm of $C$ is Gateaux differentiable at a point $f$ if and only if $|f|$ takes on its maximum at only one point, as also follows from the following evaluation of $N_{+}(x ; y)$ and $N_{-}(x ; y)$.

THEOREM 4.5. If $f(\neq 0)$ and $g$ are elements of the space $C$, then $N_{+}(f ; g)$ $=\max _{A} g(\operatorname{sign} f)$ and $N_{-}(f ; g)=\min _{A} g(\operatorname{sign} f)$, where $A$ is the set of numbers $\alpha$ for which $|f(\alpha)|=\|f\|$.

Proof. Since $N_{+}(f ; g)=\lim _{h \rightarrow+0}[\|f+h g\|-\|f\|] / h$, which is equal to $\lim _{n \rightarrow \infty}\|n f+g\|-\|n f\|$, it follows from Theorem 3.1 that $N_{+}(f ; g)$ exists. If $|f(\alpha)|=\|f\|$, then $N_{+}(f ; g) \geqq \lim _{h \rightarrow+0}[|f(\alpha)+h g(\alpha)|-|f(\alpha)|] / h=g(\alpha)[\operatorname{sign} f(\alpha)]$. Hence $N_{+}(f ; g) \geqq \max _{A} g(\operatorname{sign} f)$. Let $a$ be a limit value, as $h \rightarrow+0$, of the numbers $a_{h}$ for which $\left|f\left(a_{h}\right)+h g\left(a_{h}\right)\right|=\|f+h g\|$. Then the continuity of $\|f+h g\|$ in $h$ implies that $\lim _{h \rightarrow+0}\left|f\left(a_{h}\right)+h g\left(a_{h}\right)\right|=\|f\|$, and hence that $|f(a)|=\|f\|$. But then

$$
\frac{\|f+h g\|-\|f\|}{h}=\frac{\left|f\left(a_{h}\right)+h g\left(a_{h}\right)\right|-\|f\|}{h} \leqq \frac{\left|f\left(a_{h}\right)+h g\left(a_{h}\right)\right|-\left|f\left(a_{h}\right)\right|}{h},
$$

and if $h \rightarrow+0$ through values $h^{\prime}$ which are such that $a_{h^{\prime}} \rightarrow a$, then it is seen that $N_{+}(f ; g) \leqq \lim _{h^{\prime} \rightarrow+0} g\left(a_{h^{\prime}}\right)\left[\operatorname{sign} f\left(a_{h^{\prime}}\right)\right]=g(a)[\operatorname{sign} f(a)] \leqq \max _{A} g(\operatorname{sign} f)$. Therefore $N_{+}(f ; g)=\max _{A} g(\operatorname{sign} f)$. From this and $N_{-}(f ; g)=-N_{+}(f ;-g)$ it follows that $N_{-}(f ; g)=-\max _{A}-g(\operatorname{sign} f)=\min _{A} g(\operatorname{sign} f)$.

If we use this theorem, it follows from (6) of $\S 3$ and sign $f=|f| / f$ that if $f$ and $g$ are elements of the space $C$, then

$$
f \perp a f+g \text { if and only if } \min _{\boldsymbol{A}} g / f \leqq-a \leqq \max _{\boldsymbol{A}} g / f \text {, }
$$

where $A$ is the set of numbers $\alpha$ for which $|f(\alpha)|=\|f\|$. Thus if $\alpha \in A$, then 
$f \perp-[g(\alpha) / f(\alpha)] f+g$. Clearly orthogonality is right-unique, and the Gateaux differential $N(f ; g)$ of the norm exists, if and only if $|g(\alpha)|$ has the same value for all $\alpha \in A$ and $g(\alpha) \cdot f(\alpha)$ has the same sign for all $\alpha \in A$. If $g(\alpha)=0$ for a number $\alpha$ for which $|f(\alpha)|=\|f\|$, then $f \perp g$. This is not a necessary condition for $f \perp g$ unless or thogonality is right-unique.

THEOREM 4.6. If orthogonality is right-unique in the linear subset $T$ of the space $C$ and $f(\neq 0)$ and $g$ are elements of $T$, then $f \perp g$ if and only if $g(\alpha)=0$ for all $\alpha$ for which $|f(\alpha)|=\|f\|$.

This theorem follows easily from (7). If orthogonality is also symmetric, it can be strengthened to state that $f \perp g$ if and only if each of $|f|$ and $|g|$ is zero at all points for which the other is maximum. However, for spaces of three or more dimensions this implies the space is abstract Euclidean (Corollary 6.1).

The above results furnish means of constructing examples in which orthogonality is right-unique, left-unique, or both. Thus if $T$ is the space of all functions of the form $f=a \sin x+b\left(x-2^{-1} \pi\right)$ in the interval $(0, \pi)$, then $|f(0)|=|f(\pi)|=\|f\|$ if $|a| \leqq|b|$ and $T$ is therefore not strictly convex and orthogonality is not left-unique. The condition $\min _{A} g / f=\max _{A} g / f$ for rightuniqueness of orthogonality (7) is satisfied. For if $|a|>|b|$, then $|f|$ takes on its maximum only at one point. While if $|a| \leqq|b|$, then $|f|$ is maximum at both 0 and $\pi$. But if $g$ is any other function of the form $g=a^{\prime} \sin x$ $+b^{\prime}\left(x-2^{-1} \pi\right)$, then $g / f$ has the same value at 0 and $\pi$. Therefore orthogonality is right-unique (and additive) in $T$ and the norm of $T$ is Gateaux differentiable, although orthogonality is not left-unique and $T$ is not strictly convex.

Similarly, if $T^{\prime}$ is the space of all functions of the form $f=a \sin x+b \sin 2 x$, then orthogonality is left-unique but not right-unique. Thus $T^{\prime}$ is strictly convex, but the norm of $T^{\prime}$ is not Gateaux differentiable at all nonzero points.

Let $T^{\prime \prime}$ be the space of all functions of the form $F=a f+b g$ in the interval $(0,2)$, where

$$
f=x(2-x) \text { and } g= \begin{cases}1-x^{2} & \text { for } 0 \leqq x \leqq 1 \\ (x-2)^{2}-1 & \text { for } 1 \leqq x \leqq 2\end{cases}
$$

Then two functions belonging to $T^{\prime \prime}$ can take on their maximum at the same point only if they are multiples of each other, while no function $F$ can take on its maximum at more than one point. Thus orthogonality is both left-and right-unique, and $T^{\prime \prime}$ is strictly convex and its norm is Gateaux differentiable at all nonzero points. Then (from Theorem 4.6) it follows that $F \perp G$ if and only if $G(\alpha)=0$ for the number $\alpha$ for which $|F(\alpha)|=\|F\|$. However, it does not follow from $G$ being zero at the point for which $|F|$ is maximum that $F$ is zero at the point for which $|G|$ is maximum. Thus orthogonality is not symmetric in $T^{\prime \prime}$, and $T^{\prime \prime}$ is not an abstract Euclidean space. 
5. Hyperplanes and linear functionals. It has been shown that for any elements $x$ and $y$ of a normed linear space there can be found at least one number $a$ for which $x \perp a x+y$, and a number $b$ for which $b x+y \perp x$. The number $a$ is unique (or orthogonality is additive) if and only if the norm is Gateaux differentiable at nonzero points, while $b$ is unique if and only if the space is strictly convex (Theorems 4.1 and 4.3). Results of this type will be interpreted and extended by use of linear functionals and the concepts of conjugate spaces and supporting and tangential hyperplanes. The following definition could be given for more general surfaces than spheres [14], but is sufficient for this paper.

Definition 5.1. A hyperplane $H$ of a normed linear space $T$ is a supporting hyperplane of a sphere $S$ if the distance between $H$ and $S$ is zero and $H$ contains no interior points of $S$. If $H$ is the only supporting hyperplane which contains the boundary point $x$ of $S$, then $H$ is tangent to $S$ at $x$.

Any element $x$ of a normed linear space is orthogonal to some hyperplane through the origin, and for any hyperplane $H$ through the origin for which there exists an element $x_{0} \perp H$ there is a linear functional $f$ with $f\left(x_{0}\right)=\|f\| \cdot\left\|x_{0}\right\|$ and $f(h)=0$ for all $h \in H$ (Theorems 2.1 and 2.2). But then $f(x)=\|f\| \cdot\left\|x_{0}\right\|$ defines a hyperplane through $x_{0}$ which contains no interior points of the sphere $\|x\| \leqq\left\|x_{0}\right\|$ and is therefore a supporting hyperplane of that sphere. Hence there is a supporting hyperplane through every boundary point of a sphere $\left(^{8}\right)$. Also, if $S$ is the sphere containing all $x$ which satisfy $\|x\| \leqq\left\|x_{0}\right\|$, then $x_{0} \perp y$ if and only if $S$ has a supporting hyperplane containing $x_{0}$ and $x_{0}+y$.

The hyperplane defined by $f(x)=\left\|x_{0}\right\|$ is the only supporting hyperplane of $S$ at $x_{0}$, or also a tangent hyperplane, if and only if $f$ is the only linear functional with $\|f\|=1$ and $f\left(x_{0}\right)=\left\|x_{0}\right\|$, and then consists of all elements $x$ satisfying $f(x)=\left\|x_{0}\right\|\left({ }^{9}\right)$. But $f$ is unique if and only if there is a unique hyperplane $H$ through the origin with $x_{0} \perp H$, and hence if and only if orthogonality is right-unique or the norm is Gateaux differentiable at $x_{0}$. This tangent hyperplane is then defined by $N\left(x_{0} ; y\right)=\left\|x_{0}\right\|$, where $N\left(x_{0} ; y\right)=\lim _{h \rightarrow 0}\left[\left\|x_{0}+h y\right\|\right.$ $\left.-\left\|x_{0}\right\|\right] / h$.

THEOREM 5.1. Orthogonality is right-unique (or additive) in a normed linear space $T$ if and only if for any element $x_{0}$ with $\left\|x_{0}\right\|=1$ one of the following holds:

(1) There is a unique linear functional $f$ with $\|f\|=1$ and $f\left(x_{0}\right)=1$. This functional $f$ is then $f(y) \equiv-a \equiv N\left(x_{0} ; y\right)$, where $x_{0} \perp a x_{0}+y$.

(2) There is a hyperplane tangent to the unit sphere at the point $x_{0}$. Such a tangent hyperplane consists of all elements $y$ for which $x_{0} \perp x_{0}-y$.

Results analogous to the above can be obtained by knowing that a normed linear space is strictly convex if and only if orthogonality is left-unique (Theorem 4.3). Thus it is known that a normed linear space is strictly convex if and only if for no linear functional $f$ is there more than one point $x$ (with

(8) This also follows from Mazur [14, p. 74].

(9) Also see Mazur [15, p. 130]. 
$\|x\|=1)$ for which $f(x)=\|f\|$ - that is, no linear functional takes o1i its maximum in the unit sphere $\|x\| \leqq 1$ at more than one point. This was shown by Smulian [19, Theorem 6], but also follows easily from the theory of orthogonality:

For suppose $f$ (with $\|f\|=1$ ) takes on its maximum in the unit sphere at the two points $x_{1}$ and $x_{2}$. Then $f\left(x_{1}\right)=\left\|x_{1}\right\|=1$ and $f\left(x_{2}\right)=\left\|x_{2}\right\|=1$. Thus $f\left(x_{1}-x_{2}\right)=0$ and $f\left(x_{1}+k\left[x_{2}-x_{1}\right]\right)=1$. Hence $\left\|x_{1}+k\left(x_{2}-x_{1}\right)\right\| \geqq\left\|x_{1}\right\|=1$ for all $k$, and $x_{1} \perp\left(x_{2}-x_{1}\right)$. Likewise $x_{2} \perp\left(\dot{x}_{2}-x_{1}\right)$. Therefore $x_{1}+k\left(x_{2}-x_{1}\right) \perp\left(x_{2}-x_{1}\right)$ if $0 \leqq k \leqq 1$ (Theorem 2.3). Thus orthogonality is not left-unique and the space is not strictly convex.

Conversely, if a normed linear space is not strictly convex, then orthogonality is not left-unique. Then for some elements $x$ and $y$ and number $a$, $y \perp x$ and $a x+y \perp x$. The existence of a linear functional $f$ with $f(y)=\|f\| \cdot\|y\|$ and $f(x)=0$ follows from Theorem 2.1. But it then follows from $\|a x+y\|=\|y\|$ (Theorem 2.3) that $f(a x+y)=\|f\| \cdot\|a x+y\|$. Thus $f$ takes on its maximum in the unit sphere at the two points $y /\|y\|$ and $(a x+y) /\|a x+y\|$.

The following theorem is a consequence of the above and the fact that for every hyperplane $H$ there is a linear functional $f$ and a number $c$ such that $H$ consists of all $x$ satisfying $f(x)=c$, while the set of all $x$ satisfying an equation of the form $f(x)=c$ is a hyperplane $[14$, p. 71]

THEOREM 5.2. Orthogonality is left-unique in a normed linear space if and only if one of the following holds:

(1) No linear functional takes on its maximum in the unit sphere $\|x\| \leqq 1$ at more than one point.

(2) No supporting hyperplane of any sphere S contains more than one boundary point of $S$.

A comparison of right- and left-uniqueness of orthogonality can be made by use of conjugate spaces. Thus it has been shown by Taylor [21, Theorems 5 and 6] that a normed linear space $T$ is strictly convex if for each element $f$ of the conjugate space $T^{\prime}$ there is a unique element $F$ of $T^{\prime \prime}$ such that $\|F\|=1$ and $F(f)=\|f\|$, and that for each element $x$ of $T$ there is a unique linear functional $f$ with $\|f\|=1$ and $f(x)=\|x\|$ if $T^{\prime}$ is strictly convex. While it follows from results of Smulian [19, Theorem 8] that if every linear functional defined on $T$ attains its maximum in the unit sphere, then $T^{\prime}$ is strictly convex if the norm of $T$ is Gateaux differentiable. If these results are stated in terms of orthogonality by means of Theorems $4.1,4.3,5.1$, and 5.2 , they become the following theorem (which is not difficult to prove by the theory of orthogonality):

THEOREM 5.3. Orthogonality is left-unique in a normed linear space $T$ if it is right-unique in $T^{\prime}$, and it is right-unique in $T$ if it is left-unique in $T^{\prime}$. If every linear functional attains its maximum in the unit sphere $\|x\| \leqq 1$, then orthogonality is left-unique in $T^{\prime}$ if it is right-unique in $T$. 
The following theorems serve to complete Theorem 5.3. They make use of the concepts of weak compactness, regularity, and uniform convexity, which will be used in $\S \S 7$ and 8 to get conditions for the existence of an element orthogonal to a given closed linear subset of a normed linear space.

Definition 5.2. A sequence $\left\{x_{n}\right\}$ of elements of a normed linear space $T$ converges weakly to an element $x$ if $\lim _{n \rightarrow \infty} f\left(x_{n}\right)=f(x)$ for all linear functionals $f$ defined in $T$. A subset $S$ of $T$ is weakly compact if every sequence of elements of $S$ contains a sequence which converges weakly to an element of $S$.

THEOREM 5.4. If the unit sphere of a normed linear space $T$ is weakly compact, then orthogonality is right-unique in $T^{\prime}$ if it is left-unique in $T$.

Proof. Suppose orthogonality is not right-unique in $T^{\prime}$, and hence not additive in $T^{\prime}$ (Theorem 4.2). There then exist linear functionals $f, g$, and $h$ (defined in $T$ ) for which $f \perp g$ and $f \perp h$, but $f$ is not orthogonal to $g+h$. Thus $\|f+k g\| \geqq\|f\|$ and $\|f+k h\| \geqq\|f\|$ for all $k$, but there is a value $k_{1}$ of $k$ for which

$$
\left\|f+k_{1}(g+h)\right\|<\|f\| \text {. }
$$

Without loss of generality, $\|f\|$ can be taken as unity and $k_{1}$ to be positive. Then it follows from the discussion following Definition 4.4 that $\|f+k(g+h)\|$ $<\|f\|$ for $0<k \leqq k_{1}$. For each number $k$, choose $x_{k}$ such that $\left\|x_{k}\right\|=1, f\left(x_{k}\right) \geqq 0$, and

$$
\left|f\left(x_{k}\right)+k g\left(x_{k}\right)\right| \geqq\|f\|=1 .
$$

Likewise, for each $k$ let $y_{k}$ be such that $\left\|y_{k}\right\|=1, f\left(y_{k}\right) \geqq 0$, and $\left|f\left(y_{k}\right)+k h\left(y_{k}\right)\right|$ $\geqq\|f\|=1$. Since $\left|g\left(x_{k}\right)\right| \leqq\|g\|$ and $\left|h\left(y_{k}\right)\right| \leqq\|h\|$, it follows from $0 \leqq f\left(x_{k}\right) \leqq 1$ that $g\left(x_{k}\right) \geqq 0$ and $h\left(y_{k}\right) \geqq 0$ if $0<k<1 /\|g\|$ and $0<k<1 /\|h\|$. If $k$ satisfies these inequalities, and $k \leqq k_{1}$, then $g\left(x_{k}\right)$ and $h\left(x_{k}\right)$ must be of opposite sign, since otherwise $\left|f\left(x_{k}\right)+k\left[g\left(x_{k}\right)+h\left(x_{k}\right)\right]\right|$ would not be less than $\|f\|=1$. Likewise, $g\left(y_{k}\right)$ and $h\left(y_{k}\right)$ would also be of opposite sign. Since the unit sphere of $T$ is weakly compact, it is possible to choose sequences from the elements $x_{k}$. and $y_{k}$ which converge weakly to elements $x$ and $y$, respectively, as $k$ approaches zero. For elements $x_{k}$ and $y_{k}$ of these sequences, $f\left(x_{k}\right) \rightarrow 1$ and $f\left(y_{k}\right) \rightarrow 1$ as $k \rightarrow 0$. Thus $f(x)=f(y)=1$. Since $\|f\|=1$, it follows that $1 \leqq\|x\|$ and $1 \leqq\|y\|$. If $F$ is a linear functional for which $\|F\|=1$ and $F(x)=\|x\|$, then $\left|F\left(x_{k}\right)\right| \leqq\left\|x_{k}\right\|=1$. Hence $F(x) \leqq 1$ and $\|x\| \leqq 1$. Likewise $\|y\| \leqq 1$. Therefore $\|x\|=\|y\|=1$, and $f$ takes on its maximum in the unit sphere at both $x$ and $y$. But $g(x) \geqq 0$ and $h(x) \leqq 0$, while $g(y) \leqq 0$ and $h(y) \geqq 0$. Thus $x=y$ is possible only if $g(x)=g(y)=h(x)=h(y)=0$, and hence only if $\left|f(x)+k_{1}[g(x)+h(x)]\right|$ $=\|f\|$. But this would contradict $\left\|f+k_{1}(g+h)\right\|<\|f\|$. Since $x \neq y, f$ takes on its maximum in the unit sphere at two distinct points and orthogonality is not left-unique in $T$ (see Theorem 5.2). Therefore orthogonality is rightunique in $T^{\prime}$ if it is left-unique in $T$.

Definition 5.3. A normed linear space $T$ is regular (or reflexive) if and 
only if for every linear functional $F$ defined over the conjugate space $T^{\prime}$ there is an element $x_{0}$ of $T$ such that $F(f)=f\left(x_{0}\right)$ for all elements $f$ of $T^{\prime}$.

If the unit sphere of a normed linear space is weakly compact, then each linear functional defined in the space attains its maximum in the unit sphere. It is then possible to combine Theorems 5.3 and 5.4. However, it is known that the unit sphere of a regular Banach space is weakly compact [10, Theorem 1]. This is a stronger assumption than weak compactness, but is not greatly different since a separable Banach space is regular if its unit sphere is weakly compact $[2$, p. 189 , Theorem 13$]$.

THEOREM 5.5. If a Banach space $T$ is regular, or if the unit sphere of $T$ is weakly compact, then orthogonality is left-unique in $T^{\prime}$ if and only if it is rightunique in $T$, and it is right-unique in $T^{\prime}$ if and only if it is left-unique in $T$.

Definition 5.4. A normed linear space is uniformly convex if and only if for each $\epsilon>0$ there is a number $\delta>0$ for which $\|x\|=\|y\|=1$ and $\|x-y\|>\epsilon$ imply $\|x+y\|<2-\delta$.

It is known that a uniformly convex Banach space is regular ([16, Theorem 2] or [17]). However, a uniformly convex space is clearly strictly convex, which implies that orthogonality is left-unique.

THEOREM 5.6. If a Banach space $T$ is uniformly convex, then orthogonality is left-unique in $T$ and right-unique in $T^{\prime}$, and it is left-unique in $T^{\prime}$ if and only if it is right-unique in $T$.

It is known that if a linear functional $f$ is defined over a linear subset $S$ of a normed linear space $T$, then $f$ can be extended to all of $T$ without increasing its norm [2, p. 55, Theorem 2]. Thus for any element $x_{0}$ of $T$ there is at least one linear functional $g$ defined over $T$ and with $\|g\|=1$ and $g\left(x_{0}\right)=1$. Clearly this functional $g$ is unique if for any linear functional $f$ defined on a linear subset of $T$ there is only one such extension of $f$ to all of $T$. This condition therefore also implies that for each element $x_{0}$ there is a hyperplane tangent to the sphere $\|x\| \leqq\left\|x_{0}\right\|$ at $x_{0}$, that the norm of $T$ is Gateaux differentiable, and that orthogonality is right-unique in $T$ (see Theorems 4.2, 5.1).

Taylor has shown [21, Theorem 6] that if $T^{\prime}$ is strictly convex, then there is a unique norm-preserving extension for any linear functional defined on a linear subset of $T\left({ }^{10}\right)$. If every linear functional defined on $T$ attains its maximum in the unit sphere and orthogonality is right-unique in $T$, then $T^{\prime}$ is strictly convex (Theorem 5.3), and hence there is only one extension for any linear functional $f$ defined on a linear subset of $T$ which doesn't increase the norm of $f$. This argument is equivalent to the proof given below in terms of orthogonality:

(10) An equivalent condition is that orthogonality be left-unique in $T^{\prime}$, or that no supporting hyperplane of the unit sphere $S^{\prime}$ of $T^{\prime}$ contain more than one boundary point of $S^{\prime}$ (Theorems 4.3 and 5.2). 
THEOREM 5.7. If every linear functional defined over a normed linear space $T$ attains its maximum in the unit sphere, or if the unit sphere of $T$ is weakly compact, or if $T$ is regular or uniformly convex, then each of the following equivalent conditions is necessary and sufficient for there to be a unique norm-preserving extension over $T$ of any linear functional defined on a linear subset of $T\left(^{11}\right)$ :

(1) Orthogonality is right-unique (or additive) in $T$.

(2) The norm of $T$ is Gateaux differentiable.

(3) For any element $x_{0}$ of $T$ there is a unique linear functional $f$ with $\|f\|=1$ and $f\left(x_{0}\right)=\left\|x_{0}\right\|$.

(4) There is a tangent hyperplane at each boundary point of any sphere.

Proof. As noted above, it is known that if a normed linear space $T$ is uni- $^{-}$ formly convex or regular, or if its unit sphere is weakly compact, then every linear functional defined over $T$ attains its maximum in the unit sphere. Suppose that for a linear subset $S$ of $T$ there is a linear functional $f$ defined over $S$ which has two nonequal extensions $F$ and $G$ to all of $T$, both of which have the same norm as $f$. Then $\|F+k(F-G)\| \geqq\|F\|$ and $\|G+k(F-G)\| \geqq\|G\|$ for all $k$, and $F \perp F-G$ and $G \perp F-G$. Hence $\|F+G\|=2\|F\|=2\|G\|$ (Theorem 2.3). If $|F(x)+G(x)|=\|F+G\|$ and $\|x\|=1$, then $|F(x)|=\|F\|$ and $|G(x)|=\|G\|$ and condition (3) is not satisfied. But (1)-(4) are equivalent (Theorems 4.2 and 5.1 ), and (3) is clearly a necessary condition for the uniqueness of the normpreserving extension of any linear functional defined on a linear subset of $T$.

6. Existence of inner products in normed linear spaces. Jordan and Neumann [12] have shown that a normed linear space is abstract Euclidean if and only if $\|x+y\|^{2}+\|x-y\|^{2}=2\left[\|x\|^{2}+\|y\|^{2}\right]$ for all $x$ and $y$, while Ficken [5] has shown that an equivalent condition is that $\|a x+y\|=\|x+a y\|$ for all $a$ and $x$ and $y$ with $\|x\|=\|y\|$. These conditions were used in showing that if orthogonality as defined by either of the conditions $\|x+y\|=\|x-y\|$ or $\|x\|^{2}+\|y\|^{2}=\|x-y\|^{2}$ is either additive or homogeneous in a normed linear space $T$, then $T$ is an abstract Euclidean space $\left({ }^{12}\right)$. These types of orthogonality are symmetric in any normed linear space, while that used in this paper is not.

Birkhoff [3] has shown that a normed linear space of three or more dimensions is abstract Euclidean if and only if orthogonality (Definition 1.2) is symmetric and unique, and that this is not true for spaces of two dimensions. It has been shown that orthogonality is right-unique if and only if it is additive (Theorem 4.2), and that it is left-unique if and only if the space is strictly convex (Theorem 4.3). Thus Birkhoff's theorem can be extended to give the following:

THEOREM 6.1. Each of the following conditions is necessary and sufficient for

(11) This is similar to Taylor's theorem [21, Theorem 4], but is stronger in not assuming that the unit sphere of $T$ is weakly compact.

(12) See [11, Theorems 4.7-4.8, and 5.2-5.3]. 
a normed linear space $T$ of three or more dimensions to be an abstract Euclidean space:

(1) That orthogonality be symmetric and additive in $T$.

(2) That $T$ be strictly convex and orthogonality be symmetric in $T\left({ }^{13}\right)$.

If $T$ is a linear subset of the space $C$ of continuous functions, then a necessary and sufficient condition for orthogonality to be right-unique in $T$ is that, for any elements $f$ and $g, g / f$ have the same value at all points for which $|f|$ is maximum( $\left.{ }^{14}\right)$. This is equivalent to the condition that $g(a)=0$ for all numbers $a$ for which $|f|$ is maximum if it is zero for one such number, and is also equivalent to the condition that $f \perp g$ if and only if $g(a)=0$ for all numbers $a$ for which $|f|$ is maximum (Theorem 4.6).

COROLLARY 6.1. If $T$ is a linear subset of $C$ and is of three or more dimensions, then $T$ is an abstract Euclidean space if and only if for any nonzero elements $f$ and $g$ of $T$ it follows from $g=0$ at one point for which $|f|$ is maximum that $g=0$ at all such points, and that $f=0$ at all points for which $|g|$ is maximum.

If $S$ is any two-dimensional linear subset of a space $T$ satisfying the conditions of Corollary 6.1, then $S$ is an abstract Euclidean space and is hence equivalent to the space of functions of the form $f=a \sin x+b \cos x$ in $(0, \pi)$. However, a, two-dimensional linear subset $T$ of $C$ satisfying the conditions of Corollary 6.1 is not necessarily abstract Euclidean.

If the Gateaux differential $N(x ; y)$ of the norm exist for nonzero elements $x$ of a normed linear space $T$, then $x \perp y$ if and only if $N(x ; y)=0$ (Theorem 4.1) and orthogonality is symmetric if and only if $N(x ; y)=0$ implies $N(y ; x)$ $=0$ for nonzero elements $x$ and $y$. However, $N_{+}(x ; y)=\lim _{h \rightarrow+0}[\|x+h y\|$ - $\|x\|] / h$ exists for all elements $x$ and $y$ of a normed linear space, and it has been shown that orthogonality is symmetric and right-unique in a normed linear space if and only if $N_{+}(y, x)=0$ for any nonzero elements $x$ and $y$ satisfying $N_{+}(x ; y)=0$ (Theorem 3.5 ).

THEOREM 6.2. A normed linear space of three or more dimensions is an abstract Euclidean space if and only if $N_{+}(y ; x)=0$ for any nonzero elements $x$ and $y$ satisfying $N_{+}(x ; y)=0$.

Since symmetry of orthogonality implies the equivalence of right- and

(13) Fortet has shown that "normal projection" on a closed linear subset of $B$ is a continuous operation and that the set of all elements orthogonal to a given element of $B$ is a closed linear set if $B$ is a uniformly convex Banach space in which or thogonality is symmetric [7, Theorem II and p. 45]. Since uniform convexity implies strict convexity, his results follow easily from Theorem 6.1. Added in proof: A theorem (as yet unpublished) has been established to the effect that abstract Euclidean spaces of three or more dimensions can be characterized by symmetry of orthogonality. The assumption of additivity or of strict convexity in Theorem 6.1 is therefore unnecessary.

(14) See $(7)$ of $\$ 4$. 
left-uniqueness and hence of strict convexity and differentiability of the norm, it is interesting to consider whether orthogonality is symmetric in a strictly convex space whose norm is differentiable. Suppose $T$ is a finite-dimensional normed linear space, and that a representation of $T$ in an ordinary Euclidean space $E$ of the same number of dimensions has been constructed by means of a bilinear homeomorphism between $T$ and $E$. Let $S$ be the pseudo-sphere in $E$ which consists of all points corresponding to elements of $T$ whose norm is unity. Then $T$ is strictly convex (or orthogonality is left-unique) if and only if no supporting hyperplane of $S$ has more than one point of contact (Theorem 5.2). Also, the norm of $T$ is differentiable (or orthogonality is right-unique) if and only if $S$ has a tangent hyperplane at each point (Theorem 5.1). Clearly the convexity and symmetry of $S$ can be preserved and these conditions for uniqueness met without satisfying the condition for symmetry of orthogonality: that a line drawn through the origin parallel to a tangent hyperplane at any point $p$ of $S$ cut $S$ in points at which the tangent hyperplane is parallel to the diameter through $p$.

The conditions given above for the existence of an inner product are not sufficient for two-dimensional spaces, but the following theorems give conditions not dependent on the dimension of the space. The first of these makes use of orthogonality concepts only in the method of proof, and is similar to Ficken's condition [5] that $\|x+a y\|=\|a x+y\|$ if $\|x\|=\|y\|$.

LEMMA 6.1. If for a normed linear space $T \lim _{n \rightarrow \infty}[\|n x+y\|-\|x+n y\|]=0$ whenever $\|x\|=\|y\|$, then orthogonality is symmetric, additive, and unique in $T$ and $x \perp a x+y$ and $y \perp b y+x$ imply $a\|x\|^{2}=b\|y\|^{2}$.

Proof. Let $x$ and $y$ be elements of $T, A$ and $B$ be the algebraically smallest and largest of the numbers $a$ for which $x \perp a x+y$, and $A^{\prime}$ and $B^{\prime}$ be the algebraically smallest and largest of the numbers $b$ for which $y \perp b y+x$. If $\lim _{n \rightarrow \infty}\|n x+y\|-\|x+n y\|=0$ whenever $\|x\|=\|y\|$, then it follows from Theorem 3.1 that

$$
\lim _{n \rightarrow \infty}[\|n x+y\|-\|n x\|]=\lim _{n \rightarrow \infty}[\|x+n y\|-\|n y\|],
$$

whenever $\|x\|=\|y\|$, and that $A=A^{\prime}$. Likewise, $\|x\|=\|y\|$ implies $\lim _{n \rightarrow \infty}[\|n x-y\|-\|n x\|]=\lim _{n \rightarrow \infty}[\|x-n y\|-\|n y\|]$, and that $B=B^{\prime}$. If $x \perp y$ and $\|x\|=\|y\|$, then clearly $A \leqq 0 \leqq B$ and $A^{\prime} \leqq 0 \leqq B^{\prime}$. That $y \perp x$ now follows from Lemma 3.1. Since orthogonality is homogeneous, there was no loss of generality in assuming $\|x\|=\|y\|$. It has thus been shown that orthogonality is symmetric in any normed linear space for which $\|x\|=\|y\|$ implies $\lim _{n \rightarrow \infty}\|n x+y\|-\|x+n y\|=0$. Now suppose orthogonality is not unique in such a space. From symmetry, there are then elements $x$ and $y$ with $\|x\|=\|y\|$ and a positive number $\epsilon$ for which $x \perp a x+y$ and $a x+y \perp x$ if $|a|<\epsilon$ (Lemma 3.1). It now follows from Theorem 2.3 that $\|a x+y\|=\|y\|=\|x\|$ if $|a|<\epsilon$. Hence 


$$
\lim _{n \rightarrow \infty}[\|n x+(a x+y)\|-\|x+n(a x+y)\|]=0
$$

if $|a|<\epsilon$. But $\|x+n(a x+y)\|=\|(1+n a) x+n y\|=\|n y\|$ if $n$ is large enough that $|(1+n a) / n|<\epsilon$. Also, $\lim _{n \rightarrow \infty}[\|n x+(a x+y)\|-\|n x+y\|]=a\|x\| \quad[11$, Lemma 4.4]. Thus

$\lim _{n \rightarrow \infty}[\|n x+(a x+y)\|-\|x+n(a x+y)\|]=\lim _{n \rightarrow \infty}[\|n x+y\|-\|n y\|]+a\|x\|$,

and clearly can not be zero for all values of $a$ with $|a|<\epsilon$. Hence orthogonality is unique in $T$. Additivity of the orthogonality follows from Theorem 4.2. It has thus been shown that if $\|x\|=\|y\|$ implies $\lim _{n \rightarrow \infty}[\|n x+y\|-\|x+n y\|]$ $=0$, then it also implies $A=A^{\prime}$ and $B=B^{\prime}$, and because of the uniqueness just proved, $A=B=A^{\prime}=B^{\prime}$. Thus $x \perp a x+y$ implies $y \perp a y+x$ if $\|x\|=\|y\|$. If $\|x\| \neq\|y\|$, take $r$ such that $\|r x\|=\|y\|$. If $x \perp a x+y$ and $y \perp b y+x$, it follows from the homogeneity of orthogonality that $r x \perp(a / r)(r x)+y$ and $y \perp(b r) y$ $+r x$. Then $a / r=b r$, or $a\|x\|^{2}=b\|y\|^{2}$.

ThEOREM 6.3. A normed linear space $T$ is an abstract Euclidean space if and only if $\lim _{n \rightarrow \infty}\|n x+y\|-\|x+n y\|=0$ whenever $\|x\|=\|y\|$.

Proof. Define the inner product $(x, y)$ as $-a\|x\|^{2}$, where $x \perp a x+y$. This value of the inner product is unique because of Lemma 6.1, and it is only necessary to show that it satisfies the conditions of Definition 1.1:

(1) $(t x, y)=t(x, y)$. If $x \perp a x+y$, and $t \neq 0$, then $t x \perp(a / t)(t x)+y$ because of the homogeneity of orthogonality. Thus $(t x, y)=-(a / t)\|t x\|^{2}=-a t\|x\|^{2}$ $=t(x, y)$. If $t=0$, the proof is trivial.

(2) $(x, y)=(y, x)$. If $x \perp a x+y$, and $y \perp b y+x$, then $(x, y)=-a\|x\|^{2}$ and $(y, x)=-b\|y\|^{2}$. These are equal by Lemma 6.1.

(3) $(x, y)+(x, z)=(x, y+z)$. Suppose $x \perp a x+y$ and $x \perp b x+z$. Then $x \perp(a+b) x+(y+z)$ because of the additivity given by Lemma 6.1. Hence $(x, y)=-a\|x\|^{2},(x, z)=-b\|x\|^{2}$, and $(x, y+z)=-(a+b)\|x\|^{2}$.

(4) $(x, x)=\|x\|^{2}$. This is immediate from $x \perp(-1) x+x$.

Conversely, it follows from the definition of an inner product that for abstract Euclidean spaces $\|n x+y\|^{2}=n^{2}(x, x)+2 n(x, y)+(y, y)$ and $\|x+n y\|^{2}$ $=(x, x)+2 n(x, y)+n^{2}(y, y)$. If $\|x\|=\|y\|$, or $(x, x)=(y, y)$, then $\|n x+y\|$ $=\|x+n y\|$ for all $n$.

It has been shown (Theorem 3.5) that orthogonality is symmetric and unique in a normed linear space if $N_{+}(y ; x)=0$ for any nonzero elements $x$ and $y$ satisfying $N_{+}(x ; y)=0$. This condition can be satisfied in a normed linear space of three or more dimensions if and only if the space is abstract Euclidean (Theorem 6.2). It is possible to strengthen this and get a condition analogous to that of Theorem 6.3, valid for spaces of any number of dimensions.

THEOREM 6.4. A normed linear space $T$ is an abstract Euclidean space if and only if $N_{+}(x ;\|x\| y)=N_{+}(y ;\|y\| x)$ for all elements $x$ and $y$. 
Proof. Since $N_{+}(x ; y)=\lim _{h \rightarrow+0}[\|x+h y\|-\|x\|] / h$, it follows that

$$
\begin{aligned}
N_{+}(x ;\|x\| y)-N_{+}(y ; & \|y\| x) \\
& =\lim _{h \rightarrow+0}\left[\frac{\|(x+h y\|x\|)\|-\|x\|}{h}-\frac{\|(y+h x\|y\|)\|-\|y\|}{h}\right] \\
& =\lim _{h \rightarrow+0}\left[\frac{\|(x\|y\|+h y\|x\|)\|-\|(y\|x\|+h x\|y\|)\|}{h}\right] \\
& =\lim _{n \rightarrow \infty}[\|n(x\|y\|)+(y\|x\|)\|-\|(x\|y\|)+n(y\|x\|)\|] .
\end{aligned}
$$

But by Theorem 6.3 this is zero if and only if the space is abstract Euclidean.

7. Existence of elements orthogonal to given linear subsets of normed linear spaces. It was shown that for any $x$ there is a hyperplane $H$ with $x \perp H$, the proof depending on the fact that for any $x$ there is a linear functional $f$ with $f(x)=\|f\| \cdot\|x\|$ (Theorem 2.2). There is an analogous relation between the existence of elements orthogonal to given closed linear subsets and the existence of elements $x$ with $|f(x)|=\|f\| \cdot\|x\|$ for given linear functionals $f$.

THEOREM 7.1. A necessary and sufficient condition that there exist an element orthogonal to each closed linear subset of a normed linear space $T$ is that for each linear functional $f$ defined in $T$ there is an element $x$ with $f(x)=\|f\| \cdot\|x\|$.

Proof. If $\|f\| \neq 0$, then the set $H$ of elements for which $f=0$ is a closed linear subset. It follows from Theorem 2.1 that any element $x$ orthogonal to this set is such that $|f(x)|=\|f\| \cdot\|x\|$. Conversely, suppose $H$ is any closed linear subset of $T$. Define the functional $F$ as being zero for elements of $H$ and unity for some element $x_{0}$ not in $H$. This functional is clearly additive over the space obtained by adjoining $x_{0}$ to $H$, and its continuity follows from $H$ being closed. There is then a linear functional $f$ defined over $T$ and such that $f(x)=F(x)$ for all $x$ for which $F$ is defined [2, p. 55, Theorem 2]. If there is an element $x$ for which $f(x)=\|f\| \cdot\|x\|$, then $x \perp H$ (Theorem 2.1).

For any linear functional $f$ defined in an abstract Euclidean space $E$, there is a unique element $x$ with $\|x\|=1$ and $f(x)=\|f\|$, and for any closed linear subset $H$ in $E$ there is an element $x \perp H$ [13, pp. 11-12 and Theorem 16]. For a linear functional $f$ defined in a normed linear space with a weakly compact unit sphere there is an element $x$ for which $f(x)=\|f\| \cdot\|x\|[15$, pp. 129-130]. This is equivalent to the following theorem.

THEOREM 7.2. If the unit sphere of a normed linear space $T$ is weakly compact, then for each closed linear subset $H$ of $T$ there is at least one element $x$ for which $x \perp H$.

Definition 7.1. A functional $f(x ; y)$ is the Fréchet differential of a functional $f$ defined on a normed linear space $T$ if and only if $f(x ; y)$ is linear in $y$ and for any $\epsilon>0$ there is a positive number $\delta$, independent of $y$, for which 


$$
\left|\frac{f(x+h y)-f(x)}{h}-f(x ; y)\right|<\epsilon\|y\|
$$

if $|h|<\delta ; f(x ; y)$ is a uniform Fréchet differential if the number $\delta$ can be chosen independently of $x$ for $\|x\|=1$.

It is known that a Banach space is regular if its norm is uniformly Fréchet differentiable $[20$, p. 648], while the unit sphere of a regular Banach space is weakly compact [10, Theorem 1$]$. Thus either of these conditions could be used in place of the weak compactness of Theorem 7.2. Fréchet differentiability of the norm has the added advantage of implying Gateaux differentiability, which will be directly or implicitly needed in the evaluation of linear functionals.

THEOREM 7.3. If the Banach space $B$ is regular, or if the norm of $B$ is uniformly Fréchet differentiable, then for each closed linear subset $H$ of $B$ there is at least one element $x$ for which $x \perp H$. If $f$ is a linear functional defined in $B$, then there is at least one element $x$ for which $f(x)=\|f\| \cdot\|x\|$.

It is known that a uniformly convex Banach space is regular [16, Theorem 2]. Furthermore, a uniformly convex space is clearly strictly convex, and hence for a linear functional $f$ defined in a uniformly convex Banach space $B$ there is not more than one element $x$ with $\|x\|=1$ and $f(x)=\|f\|[19$, Theorem 6]. Therefore there is not more than one element on a given side of a hyperplane $H$ through the origin and orthogonal to $H$, and Theorem 7.3 becomes $\left({ }^{15}\right)$ :

THEOREM 7.4. If a Banach space $B$ is uniformly convex, then on each side of a hyperplane $H$ through the origin there is a unique element $x$ with $\|x\|=1$ and $x \perp H$. If $f$ is a linear functional defined on $B$, then there is a unique element $x$ with $\|x\|=1$ and $f(x)=\|f\|$.

8. General forms for linear functionals. If $f$ is a linear functional defined in a complete abstract Euclidean space, then there is one and only one element $x_{0}$ such that $f(y) \equiv\left(x_{0}, y\right)$, where $\left(x_{0}, y\right)$ is the inner product of $x_{0}$ and $y$ $\left[13\right.$, Theorem 11]. Then $f\left(x_{0}\right)=\|f\|_{1}\left\|x_{0}\right\|$, and $f$ takes on its maximum in the unit sphere only at the point $x_{0} /\left\|x_{0}\right\|$, while $x_{0}$ is orthogonal to the hyperplane $H$ of all $h$ satisf ying $\left(x_{0}, h\right)=0$. Also, for abstract Euclidean spaces,

$$
N\left(x_{0} ; y\right)=\frac{\left(x_{0}, y\right)}{\left\|x_{0}\right\|}=-a\left\|x_{0}\right\|,
$$

where $N\left(x_{0} ; y\right)$ is the Gateaux differential of the norm at $x_{0}$ and $x_{0} \perp a x_{0}+y$ (Theorem 4.1). Thus for any linear functional $f$ with $\|f\|=1$ there is a unique

(15) See the discussion preceding Theorem 5.2. Two points are on the same side of a hyperplane $H$ if the line joining them does not intersect $H$. Theorem 7.4 would also follow from a theorem of Fortet [7, Theorem 1]. 
element $x_{0}$ for which $f(y)$ is identically equal to $N\left(x_{0} ; y\right),\left(x_{0}, y\right) /\left\|x_{0}\right\|$, or $-a\left\|x_{0}\right\|$.

If there is at least one element orthogonal to any given closed linear subset of a normed linear space, then for any linear functional $f$ with $\|f\|=1$ there is an element $x$ with $f(x)=1$ and $\|x\|=1$ (Theorem 7.1). If there is no hyperplane $H$ through the origin with two linearly independent elements orthogonal to $H$, then this element $x$ is unique. But if no element is orthogonal to more than one hyperplane through the origin, then orthogonality is additive and $x \perp a x+y$ implies $f(a x+y)=0$ :

THEOREM 8.1. If there is at least one element orthogonal to any given closed linear subset of a normed linear. space $T$ and no element of $T$ is orthogonal to more than one hyperplane through the origin, then for a linear functional $f d e-$ fined in $T$ and with $\|f\|=1$ there is at least one element $x_{0}$ such that

$$
f(y) \equiv-a\left\|x_{0}\right\| \text {, where } x_{0} \perp a x_{0}+y .
$$

There is only one such element $x_{0}$ having $\left\|x_{0}\right\|=1$ if no two linearly independent elements are orthogonal to the same hyperplane through the origin.

Equivalent conditions for the uniqueness of the element $x_{0}$ with $\left\|x_{0}\right\|=1$ and $f(y) \equiv-a\left\|x_{0}\right\|$ are: (1) that orthogonality be left-unique; (2) that no supporting hyperplane of the unit sphere contain more than one boundary point; and (3) that $T$ be strictly convex (see Theorems 4.3 and 5.2).

If no element of $T$ is orthogonal to more than one hyperplane through the origin, then the norm of $T$ is Gateaux differentiable (Theorems 4.1 and 5.1). Thus any linear functional $f$ with $f(y) \equiv-a\left\|x_{0}\right\|$, as above, is also equal to the differential $N\left(x_{0} ; y\right)$. There is at least one element orthogonal to any closed linear subset of a Banach space $B$ if $B$ is regular, or if the unit sphere of $B$ is weakly compact (Theorems 7.2-7.3). Thus either of these conditions could have been used in Theorem 8.1 if $T$ had been taken as a Banach space. However, it should be noted that they are equivalent, since a Banach space is regular if its unit sphere is weakly compact and its norm is differentiable [19, Theorem 10], while the unit sphere of a regular Banach space is weakly compact [10, Theorem 1].

Uniform Fréchet differentiability of the norm is a stron'er condition, which also implies that there is at least one element orthogonal to any given closed linear subset (Theorem 7.3). Hence if the norm of a Banach space is uniformly Frêchet differentiable, then for a linear functional $f$ with $\|f\|=1$ there is at least one element $x_{0}$ such that $f(y) \equiv N\left(x_{0} ; y\right) \equiv-a\left\|x_{0}\right\|$, where $x_{0} \perp a x_{0}+y$ and $N\left(x_{0} ; y\right)$ is the differential of the norm at $x_{0}$.

If it is assumed that the space is uniformly convex, then the uniqueness of $x_{0}$ follows from the resulting strict convexity and Theorem 7.4.

THEOREM 8.2. If $B$ is a uniformly convex Banach space whose norm is Gateaux differentiable at all nonzero points, then for a linear functional $f$ with 
$\|f\|=1$ there is one and only one element $x_{0}$ such that $\left\|x_{0}\right\|=1$ and $f(y) \equiv N\left(x_{0} ; y\right)$ $\equiv-a$, where $x_{0} \perp a x_{0}+y$ and $N\left(x_{0} ; y\right)$ is the differential of the norm at $x_{0}$.

It is known that the conclusion of Theorem 8.2 is valid in abstract Euclidean spaces [13, Theorem 11], as would also follow from the fact that an abstract Euclidean space is uniformly convex and its norm is differentiable. However, it is possible for a normed linear space to satisfy the conditions of Theorem 8.2 without being abstract Euclidean. This is shown by the following examples, which also furnish illustrations of other principles of orthogonality.

EXAMPLE 8.1. Consider the Banach space $l^{(r)}(r \geqq 1)$ of all sequences $x=\left(x_{1}, x_{2}, \cdots\right)$ for which $\sum_{i=1}^{\infty}\left|x_{i}\right| r$ is convergent, where $\|x\| r=\sum_{i=1}^{\infty}\left|x_{i}\right| r$.

Any linear functional $f$ with $\|f\|=1$ defined in $l^{(1)}$ can be written in the form $f(y)=\sum_{i=1}^{\infty} C_{i} y_{i}$, where the least upper bound of $\left|C_{i}\right|$ is $1[2$, p. 67]. Since $\|x\|=\sum_{i=1}^{\infty}\left|x_{i}\right|$ if $x \in l^{(1)}$, it follows that $f(x)=\|x\|$ implies $C_{i} x_{i}=\left|x_{i}\right|$ for each $i$. Hence any linear functional $f$ defined in $l^{(1)}$ and having $\|f\|=1$ and $f(x)=\|x\|$ is of the form

$$
f(y)=\sum_{i}^{x_{i} \neq 0} \frac{x_{i}}{\left|x_{i}\right|} y_{i}+\sum_{i}^{x_{i}=0} C_{i} y_{i},
$$

where $\left|C_{i}\right| \leqq 1$ for each $i$. For any such linear functional $f$, the set of elements $y$ satisfying $f(y)=1$ is a supporting hyperplane of the unit sphere at the point $x /\|x\|$ (see Definition 5.1 and the following discussion). It follows from Theorem 2.1 that $x \perp a x+y$ if and only if for each $i$ for which $x_{i}=0$ a number $C_{i}$ with $\left|C_{i}\right| \leqq 1$ can be chosen such that $f(a x+y)=a\|x\|$ $+\sum_{i}^{x_{i} \neq 0}\left(x_{i} /\left|x_{i}\right|\right) y_{i}+\sum_{i}^{x_{i}=0} C_{i} y_{i}=0$, or if and only if

$$
\sum_{i}^{x_{i} \neq 0} \frac{x_{i}}{\left|x_{i}\right|} y_{i}-\sum_{i}^{x_{i}=0}\left|y_{i}\right| \leqq-a\|x\| \leqq \sum_{i}^{x_{i} \neq 0} \frac{x_{i}}{\left|x_{i}\right|} y_{i}+\sum_{i}^{x_{i}=0}\left|y_{i}\right| \text {. }
$$

Thus orthogonality is right-unique, or additive, at an element $x=\left(x_{1}, x_{2}, \cdots\right)$ of the space $l^{(1)}$ if and only if $x_{i} \neq 0$ for any $i$. This condition is also necessary and sufficient for the existence of a tangent hyperplane at the point $x /\|x\|$ of the unit sphere (Theorem 5.1), and for the existence of the Gateaux differential of the norm at $x$, it following from (6) of $\S 3$ that

$N_{+}(x ; y)=\sum_{i}^{\alpha_{i} \neq 0} \frac{x_{i}}{\left|x_{i}\right|} y_{i}+\sum_{i}^{x_{i}=0}\left|y_{i}\right|$ and $N_{-}(x ; y)=\sum_{i}^{x_{i} \neq 0} \frac{x_{i}}{\left|x_{i}\right|} y_{i}-\sum_{i}^{x_{i}=0}\left|y_{i}\right|$.

If $r>1$, then any linear functional $f$ defined in $l^{(r)}$ and having $\|f\|=1$ is of the form $[2$, p. 68]

$$
f(y)=\sum_{i=1}^{\infty} C_{i} y_{i}, \text { where } \sum_{i=1}^{\infty}\left|C_{i}\right| \cdot=1 \text { and } s=\frac{r}{r-1} .
$$

If $C_{i}=x_{i}\left|x_{i}\right|^{r-2} /\|x\|^{r-1}$, then $f(x)=\|x\|$. It will follow that these are the only 
possible values of $C_{i}$ for which $f(x)=\|x\|$ if it is shown that orthogonality is right-unique, since there could then only be one linear functional $f$ with $\|f\|=1$ and $f(x)=\|x\|$ (Theorem 5.1) $\left({ }^{16}\right)$.

By definition, $x \perp y$ if and only if

$$
\|x+h y\|-\|x\|=\left[\sum_{i=1}^{\infty}\left|x_{i}+h y_{i}\right| r\right]^{1 / r}-\left[\sum_{i=1}^{\infty}\left|x_{i}\right| r\right]^{1 / r} \geqq 0 \quad \text { for all } h,
$$

or $\sum_{i=1}^{\infty}\left[\left|x_{i}+h y_{i}\right|^{r}-\left|x_{i}\right| r\right] \geqq 0$ for all $h$. Thus if $x \perp y$, then

$$
\lim _{h \rightarrow+0}\left[\sum_{i=1}^{\infty} \frac{\left|x_{i}+h y_{i}\right| r-\left|x_{i}\right| r}{h}\right] \geqq 0 .
$$

It can be shown by using the convergence of $\sum_{i=1}^{\infty}\left|x_{i}\right|^{r}$ and $\sum_{i=1}^{\infty}\left|y_{i}\right|^{r}$ that this is equal to

$$
\sum_{i=1}^{\infty}\left[\lim _{h \rightarrow+0} \frac{\left|x_{i}+h y_{i}\right| r-\left|x_{i}\right| r}{h}\right]=\sum_{i=1}^{\infty} r\left|x_{i}\right|^{r-2}\left(x_{i} y_{i}\right) .
$$

Hence $x \perp y$ implies $\sum_{i=1}^{\infty}\left|x_{i}\right|^{r-2}\left(x_{i} y_{i}\right) \geqq 0$. Putting $(a x+y)$ and $-(a x+y)$ in place of $y$ in this shows that $x \perp a x+y$ implies $a\|x\| r+\sum_{i=1}^{\infty}\left|x_{i}\right|^{r-2}\left(x_{i} y_{i}\right)=0$, and hence that the number $a$ for which $x \perp a x+y$ (Corollary 2.2) is unique. Thus orthogonality is right-unique in $l^{(r)}(r>1)$ and for any elements $x$ and $y$

$$
x \perp-\left[\sum_{i=1}^{\infty} \frac{\left|x_{i}\right|^{r-2}\left(x_{i} y_{i}\right)}{\|x\|^{r}}\right] x+y .
$$

It now follows that the above values of the numbers $C_{i}$ are the only values for which $f(x)=\|x\|$, and from this that there is a tangent hyperplane at each boundary point of the unit sphere in $l^{(r)}(r>1)$ and that the norm of $l^{(r)}(r>1)$ is Gateaux differentiable [Theorems 4.1 and 5.1]. If this differential of the norm is $N(x ; y)$, then

$$
f(y) \equiv N(x ; y) \equiv \sum_{i=1}^{\infty} \frac{\left|x_{i}\right|^{r-2}\left(x_{i} y_{i}\right)}{\|x\|^{r-1}}
$$

is the only linear functional with $\|f\|=1$ and $f(x)=\|x\|$. It is clear from this that for any linear functional $f$ defined in $l^{(r)}(r>1)$ there is one and only one element $x$ with $\|x\|=1$ and $f(x)=\|x\|$. Thus no supporting hyperplane of any sphere $S$ of $l^{(r)}(r>1)$ contains more than one boundary point of $S$, orthogonality is left-unique, and $l^{(r)}(r>1)$ is strictly convex (Theorems 4.3 and 5.2).

However, the space $l^{(r)}(r>1)$ is not only strictly convex, but is uniformly convex [4]. By means of this fact, a proof of the general form of linear functionals in $l^{(r)}(r>1)$ can be given by means of the theory of orthogonality:

(16) That only one such linear functional $f$ can exist for an element $x$ of $l(r)(r>1)$ is shown by Mazur [14, p. 79]. 
Since it was shown above that the norm of $l^{(r)}$ is Gateaux differentiable, it follows from Theorem 8.2 that for any linear functional $f$ with $\|f\|=1$ there is a unique element $x_{0}=\left(x_{1}, x_{2}, \cdots\right)$ with $\left\|x_{0}\right\|=1$ and $f(y) \equiv-a$, where $x_{0} \perp a x_{0}+y$. But because of the above evaluation of the number $a$, this is $f(y) \equiv \sum_{i=1}^{\infty}\left|x_{i}\right|^{r-2}\left(x_{i} y_{i}\right)$. Thus every linear functional defined in $l^{(r)}(r>1)$ can be written in this form, or in the equivalent form below, if $\|f\|=1$ :

$$
f(y) \equiv \sum_{i=1}^{\infty} C_{i} y_{i}, \quad \text { where } \sum_{i=1}^{\infty}\left|C_{i}\right|^{s}=1 \text { and } s=\frac{r}{r-1} .
$$

EXAmple 8.2. Consider the Banach space $L^{(r)}(r \geqq 1)$ of all functions defined in $(0,1)$ whose $r$ th power is Lebesque in tegrable, where $\|f\|^{r}=\int_{0}^{1}|f(t)| r d t$.

The theory of orthogonality can be applied to such spaces in a way completely analogous to that of Example 8.1. If $r=1$, then $f \perp a f+g$ if and only if

$$
\int_{A} \frac{f g}{|f|} d t-\int_{B}|g| d t \leqq-a\|f\| \leqq \int_{A} \frac{f g}{|f|} d t+\int_{B}|g| d t,
$$

where $A$ and $B$ are the sets of values of $t$ for which $f(t) \neq 0$, and for which $f(t)=0$, respectively. Thus

$$
N_{+}(f ; g)=\int_{A} \frac{f g}{|f|} d t+\int_{B}|g| d t \quad \text { and } \quad N_{-}(f ; g)=\int_{A} \frac{f g}{|f|} d t-\int_{B}|g| d t \text {, }
$$

and the norm of $L^{(1)}$ is Gateaux differentiable at $f$ if and only if $B$ is of measure zero.

By a method analogous to that of Example 8.1, it can be shown that orthogonality is right-unique in $L^{(r)}(r>1)$ and that $\left.{ }^{17}\right)$

$$
f \perp-\left[\|f\|^{-r} \int_{0}^{1}|f|^{r-2}(f g) d t\right] f+g, \quad \text { or } \quad N(f ; g)=\|f\|^{1-r} \int_{0}^{1}|f|^{r-2}(f g) d t .
$$

Thus the norm of $L^{(r)}$ is Gateaux differentiable. Since $L^{(r)}$ is uniformly convex [4], it follows from Theorem 8.2 that if $F$ is any linear functional defined over $L^{(r)}$ and having $\|F\|=1$, then there is a unique element $f_{0}$ of $L^{(r)}$ for which $\left\|f_{0}\right\|=1$ and

$$
F(g) \equiv \int_{0}^{1}\left|f_{0}\right|^{r-2}\left(f_{0} g\right) d t .
$$

Thus every linear functional $F$ defined in $L^{(r)}(r>1)$ and having $\|F\|=1$ is of this form, or the equivalent form $\left({ }^{18}\right)$ :

$$
F(g) \equiv \int_{0}^{1} C(t) g(t) d t, \quad \text { where } \quad \int_{0}^{1}|C|^{s} d t=1 \quad \text { and } \quad s=\frac{r}{r-1} .
$$

(17) This evaluation of $N(f ; g)$ is obtained by Mazur [15] in a different way.

$\left.{ }^{18}\right)$ This form for linear functionals in $L^{(r)}(r>1)$ is given by Banach [2, p. 64]. 


\section{REFERENCES}

1. G. Ascoli, Sugli spazi lineari metrici e le loro varieta lineari, Annali di Matematica Pura ed Applicata (4) vol. 10 (1932) pp. 33-81.

2. S. Banach, Théorie des operations linéaires, Warsaw, 1932.

3. G. Birkhoff, Orthogonality in linear metric spaces, Duke Math. J. vol. 1 (1935) pp. 169172. 414.

4. J. A. Clarkson, Uniformly convex spaces, Trans. Amer. Math. Soc. vol. 40 (1936) pp. 396-

5. F. A. Ficken, Note on the existence of scalar products in normed linear spaces, Ann. of Math. vol. 45 (1944) pp. 362-366.

6. R. Fortet, Remarques sur les espaces uniformément convexes, C. R. Acad. Sci. Paris vol. 210 (1940) pp. 497-499.

7. - Remarques sur les espaces uniformément convexes, Bull. Soc. Math. France vol. 69 (1941) pp. 23-46.

8. M. Frechet, Sur la notion de differentielle, J. Math. Pures Appl. vol. 16 (1937) pp. 233250.

9. - Sur la definition axiomatique d'une classe d'espaces vectoriels distancies applicables vectoriellement sur l'espaces de Hilbert, Ann. of Math. vol. 36 (1935) pp. 705-718.

10. V. Gantmakher and V. Smulian, Sur les espaces lineaires dont la sphere unitaire est faiblement compacte, C. R. (Doklady) Acad. Sci. URSS. vol. 17 (1937) pp. 91-94.

11. R. C. James, Orthogonality in normed linear spaces, Duke Math. J. vol. 12 (1945) pp. 291-302.

12. P. Jordan and J. von Neumann, Inner products in linear, metric spaces, Ann. of Math. vol. 36 (1935) pp. 719-723.

13. H. Löwig, Komplexe Euklidische Räume von beliebiger endlicher oder transfiniter Dimensionzahl, Acta Litterarum Ac Scientiarum vol. 7 (1934-1935) pp. 1-33.

14. S. Mazur, Über convexe Mengen in linearen normierten Räumen, Studia Mathematica vol. 4 (1933) pp. 70-84.

15. - Über schwache Konvergenz in den Räumen ( $\left.L^{p}\right)$, Studia Mathematica vol. 4 (1933) pp. 128-133.

16. D. Milman, On some criteria for the regularity of spaces of the type (B), C. R. (Doklady) Acad. Sci. URSS. vol. 20 (1938) pp. 243-246.

17. B. J. Pettis, $A$ proof that every uniformly convex space is reflexive, Duke Math. J. vol. 5 (1939) pp. 249-253.

18. B. D. Roberts, On the geometry of abstract vector spaces, Tôhoku Math, J. vol. 39 (1934) pp. 42-59.

19. V. Smulian, On some geometrical properties of the unit sphere in the space of the type (B), Rec. Math. (Mat. Sbornik) N. S. vol. 48 (1938) pp. 90-94.

20. — Sur la derivabilité de la norme dans l'espace de Banach, C. R. (Doklady) Acad. Sci. URSS. vol. 27 (1940) pp. 643-648. 547.

21. A. E. Taylor, The extension of linear functionals, Duke Math. J. vol. 5 (1939) pp. 538-

22. - Derivatives in the calculus, Amer. Math. Monthly vol. 49 (1942) pp. 631-642.

California Institute of Technology, Pasadena, Calif. 